\title{
Semi-empirical catalog of early-type galaxy-halo systems: dark matter density profiles, halo contraction and dark matter annihilation strength
}

\author{
Kyu-Hyun Chae, ${ }^{a, b}$ Andrey V. Kravtsov, ${ }^{c, d}$ Joshua A. Frieman ${ }^{b, c, d}$ \\ Mariangela Bernardi ${ }^{e}$ \\ ${ }^{a}$ Department of Astronomy and Space Science, Sejong University, 98 Gunja-dong Gwangjin- \\ $\mathrm{Gu}$, Seoul 143-747, Republic of Korea \\ ${ }^{b}$ Center for Particle Astrophysics, Fermi National Accelerator Laboratory, P.O. Box 500, \\ Batavia, IL 60510, USA \\ ${ }^{c}$ Kavli Institute for Cosmological Physics, 5640 South Ellis Avenue, The University of \\ Chicago, Chicago, IL 60637, USA \\ ${ }^{d}$ Department of Astronomy and Astrophysics, 5640 South Ellis Avenue, The University of \\ Chicago, Chicago, IL 60637, USA \\ ${ }^{e}$ Department of Physics and Astronomy, University of Pennsylvania, 209 South 33rd Street, \\ Philadelphia, PA 19104, USA
}


E-mail: chae@sejong.ac.kr, andrey@oddjob.uchicago.edu,frieman@fnal.gov, bernardm@physics.upenn.edu

Abstract. With Sloan Digital Sky Survey galaxy data and halo data from up-to-date Nbody simulations within the $\Lambda$ CDM framework we construct a semi-empirical catalog (SEC) of early-type galaxy-halo systems by making a self-consistent bivariate statistical match of stellar mass $\left(M_{\star}\right)$ and velocity dispersion $(\sigma)$ with halo virial mass $\left(M_{\text {vir }}\right)$ as demonstrated here for the first time. We then assign stellar mass profile and velocity dispersion profile parameters to each system in the SEC using their observed correlations with $M_{\star}$ and $\sigma$. Simultaneously, we solve for dark matter density profile of each halo using the spherical Jeans equation. The resulting dark matter density profiles deviate in general from the dissipationless profile of Navarro-Frenk-White or Einasto and their mean inner density slope and concentration vary systematically with $M_{\mathrm{vir}}$. Statistical tests of the distribution of profiles at fixed $M_{\text {vir }}$ rule out the null hypothesis that it follows the distribution predicted by dissipationless N-body simulations for $M_{\mathrm{vir}} \lesssim 10^{13.5-14.5} \mathrm{M}_{\odot}$. These dark matter profiles imply that dark matter density is, on average, enhanced significantly in the inner region of halos with $M_{\text {vir }} \lesssim 10^{13.5-14.5} \mathrm{M}_{\odot}$ supporting halo contraction. The main characteristics of halo contraction are: (1) the mean dark matter density within the effective radius has increased by a factor varying systematically up to $\approx 3-4$ at $M_{\text {vir }}=10^{12} \mathrm{M}_{\odot}$, and $(2)$ the inner density slope has a mean of $\langle\alpha\rangle \approx 1.3$ with $\rho_{\mathrm{dm}}(r) \propto r^{-\alpha}$ and a halo-to-halo rms scatter of $\operatorname{rms}(\alpha) \sim 0.4-0.5$ for $10^{12} \mathrm{M}_{\odot} \lesssim M_{\text {vir }} \lesssim 10^{13-14} \mathrm{M}_{\odot}$ steeper than the NFW profile $(\alpha=1)$. Based on our results we predict that halos of nearby elliptical and lenticular galaxies can, in principle, be promising targets for $\gamma$-ray emission from dark matter annihilation.

Keywords: galaxy dynamics, galaxy formation, dark matter experiments

ArXiv ePrint: 1202.2716 


\section{Contents}

1 Introduction $\quad 1$

2 Data: galaxies and dark halos 3

2.1 Observed galaxies from the SDSS 3

2.2 Theoretical halos from the Bolshoi dark matter only simulation 3

3 Statistically matching galaxies with halos: a bivariate log-normal distribution of stellar mass and velocity dispersion as a function of halo mass for early-type systems

3.1 The detailed procedure

3.2 The result: a semi-empirical catalog of early-type galaxy-halo systems with some parameters undetermined

4 Jeans dynamical modeling of early-type galaxy-halo systems

4.1 The spherical Jeans equation

4.2 Models for the dark matter distribution

4.3 Results on dark matter density profiles

4.3.1 Restricted results based only on the SDSS velocity dispersion

4.3.2 Dark matter density profiles statistically matched with velocity dispersion profiles: completion of the semi-empirical catalog

5 Implication for baryon-induced halo contraction and characterization of the contracted profiles

6 Characterizing dark matter annihilation strength in the halos of early-type galaxies

7 Conclusions

A SDSS early-type galaxy parameter correlations

B An integral solution of the Spherical Jeans equation

C Effects of varying anisotropies of velocity dispersions

D Dynamical mass scaling relations and alternative results for halo mass profile

\section{Introduction}

The currently standard cold dark matter (to be referred to as $\Lambda \mathrm{CDM}$ in conjunction with Einstein's cosmological constant $\Lambda$ ) model of structure formation requires that galaxies form at the centers of dark matter halos $[1,2]$. Dark matter halos embedding galaxies are indicated by rotation curves of spiral galaxies [3-5], velocity dispersions of early-type (i.e. elliptical and lenticular) galaxies [6-8], and gravitational lensing effects induced by galaxies [9-11] 
and galaxy clusters $[12,13]$. In the $\Lambda \mathrm{CDM}$ model initial small density fluctuations grow by gravity and overdense regions collapse to form halos dominated by CDM and halos merge subsequently to form larger and larger halos in a hierarchical fashion. These halos provide gravitational potential wells for baryonic matter to sink dissipationally to form visible galaxies in their centers. Current $\Lambda$ CDM-based galaxy formation models reproduce well observed clustering properties of galaxy distributions and are ever improving towards the goal of explaining all observed statistical and intrinsic properties of galaxies [14-16].

Despite the fact that dark matter halos are pivotal for the standard $(\Lambda \mathrm{CDM})$ model of structure formation to explain observed galaxies, our current knowledge of them is limited in two main aspects: dark matter particles have never been identified conclusively [17-19] and statistical properties of dark matter distribution within halos have not been characterized fully and robustly through observational studies [20-23], hydrodynamic simulations [24-28] or empirical modeling of galaxy formation [29-31]. In particular, detailed dark matter density profiles including both inner density slope and concentration have been obtained only for a limited number of individual systems [32-37]. Precise knowledge of the dark matter distribution within halos is crucial for the search of dark matter particles because both direct and indirect detections of dark matter particles depend on the dark matter density within halos $[19,38]$. The dark matter distribution within halos is expected to be modified by dissipational galaxy formation in their centers from the initial distribution predicted by dissipationless N-body simulations $[24-27,39,40]$. Hence, a precise statistical characterization of dark matter distribution is crucial for galaxy formation physics as well [24, 25]. Galaxy formation modeling requires properly taking into account halo contraction effects. This means that observationally determined contracted halo mass profiles provide useful constraints on galaxy formation physics including dissipational gas cooling/accretion, star formation/feedback, supernovae and AGN.

Here we present a procedure of constructing a semi-empirical catalog of early-type galaxies and their embedding halos. We intend to construct a realistic catalog that is useful for simulational studies such as lensing and at the same time produce a rigorous and general statistical characterization of halo mass profiles that is allowed by currently best statistical knowledge of galaxies and halos through a Jeans analysis. Our approach is to combine a statistically representative sample of observed galaxies with halos from a cosmological dissipationless N-body simulation. Specifically, we have two complete and separate sets of data for galaxies and halos: photometric and spectroscopic data for galaxies [41, 42] from the Sloan Digital Sky Survey (SDSS) [43] and halos from the Bolshoi simulation [44] (section 2). Based on the data sets we follow two steps to infer the dark matter distribution in halos. The first step is to make a statistically consistent one-to-one match between galaxies and halos for early-type systems by simultaneously assigning stellar masses $\left(M_{\star}\right)$ and velocity dispersions $(\sigma)$ to halo virial masses $\left(M_{\text {vir }}\right)$ so that observed statistical functions (of $M_{\star}$ and $\sigma$ ) and correlations (between $M_{\star}$ and $\sigma$ and between $M_{\star}$ and $M_{\text {vir }}$ ) are reproduced (section 3). Each galaxy with $M_{\star}$ and $\sigma$ assigned is further assigned the effective radius $\left(R_{\mathrm{e}}\right)$ and the Sérsic index $(n)$ of the light distribution using their observed correlations with $M_{\star}$ and $\sigma$ (appendix A). We refer to the resulting catalog as a semi-empirical catalog (SEC) of early-type galaxy-halo systems. The next step is to carry out Jeans dynamical modeling of each system with the assigned galaxy parameters and the unknown dark matter distribution (section 4). Based on the stellar mass profile and the SDSS velocity dispersion we can obtain only a degenerate set (i.e. a range) of dark matter density profiles for each system and we show some restricted results under special assumptions (section 4.3.1). But, then using 
observational constraints on velocity dispersion profiles (VPs) we assign a VP to each galaxy and simultaneously a general-class dark matter density profile to the embedding halo using the spherical Jeans equation so that the posterior distribution of VPs matches the observed distribution (section 4.3.2). We test our results against physically and observationally wellmotivated dynamical constraints (appendix D). We discuss the implications of our results for halo contraction (section 5) and dark matter annihilation strength (section 6). We conclude in section 7 .

\section{Data: galaxies and dark halos}

According to the $\Lambda \mathrm{CDM}$ paradigm a galaxy is embedded near the center of its host halo. The halo itself may be embedded in a larger halo or may embed smaller halos referred to as subhalos. The larger host halo and the subhalos also embed galaxies at their centers. In this way one can identify a one-to-one match between galaxies (including satellites) and halos (including subhalos) [15, 45-50]. A reliable match requires unbiased samples of galaxies and halos.

\subsection{Observed galaxies from the SDSS}

A large and unbiased galaxy sample is provided by the completed SDSS. This survey covers a large area of sky providing a low redshift galaxy sample unaffected by large scale structures of the Universe [43]. Galaxies are observed not only photometrically but also spectroscopically providing structural and kinematical parameters such as the total stellar mass $M_{\star}$, the stellar velocity dispersion $\sigma$, the effective radius $R_{\mathrm{e}}$, [41] and the Sérsic index $n$ [42]. The velocity dispersion $\sigma$ refers to the luminosity-weighted line-of-sight velocity dispersion (LOSVD) within $R_{\mathrm{e}} / 8$ as described in [41]. Velocity dispersions are actually measured within a fixed aperture of radius 1.5 arcsec. The fixed aperture corresponds to different physical scales that are smaller than $R_{\mathrm{e}}$ for most galaxies. Measured velocity dispersions are then corrected to a fixed physical radius of $R_{\mathrm{e}} / 8$ using a typically observed radial profile of luminosity-weighted LOSVDs within $R_{\mathrm{e}}$. This corrected velocity dispersion thus corresponds to the luminosityweighted LOSVD within $R_{\mathrm{e}} / 8$.

Galaxies can be classified by eye inspection, color, spectral features, or luminosity profile. None of these methods are perfect in separating galaxies by morphology. With the goal of dynamical modeling we select a galaxy population that consist mostly of early-type spheroidal systems which are predominantly velocity dispersion supported systems. Our selection is based on a luminosity profile concentration index $C_{r}$, which is the ratio of the radial scale which contains 90 percent of the Petrosian luminosity in the $r$-band to that which contains 50 percent. Spheroidal (early-type) galaxies are known to have more concentrated profiles compared with disk (late-type) galaxies. We use the criterion of $C_{r}>2.86$ to select spheroidal galaxies [41]. Our working assumption is that for our selected galaxies any rotating disks, if present, can be ignored for the Jeans dynamical modeling.

\subsection{Theoretical halos from the Bolshoi dark matter only simulation}

Cosmological N-body simulations can be used to produce samples of dark matter halos. The predicted statistics of these halos without galaxies are robust classical results of cosmological physics. The predicted statistical properties of halos, however, depend on the adopted values of cosmological parameters. We use the Bolshoi simulation [44] that is based on the following up-to-date cosmological parameters of the flat $\Lambda \mathrm{CDM}$ cosmology: $h=0.7, \Omega_{\mathrm{m}}=0.27$, 
$n_{s}=0.95$ and $\sigma_{8}=0.82$ consistent with Wilkinson Microwave Anisotropy Probe seven-year results [51]. The Bolshoi simulation differs from the Millennium simulation [14] particularly in that the latter adopts a higher $\sigma_{8}=0.9$. The Bolshoi simulation uses $2048^{3}$ particles in a cosmological box of size $250 \mathrm{~h}^{-1} \mathrm{Mpc}$ allowing a statistically representative complete catalog of halos with virial mass (defined below) $1.5 \times 10^{10} h^{-1} \mathrm{M}_{\odot}<M_{\text {vir }}<(1-2) \times 10^{15} h^{-1} \mathrm{M}_{\odot}$.

The Bolshoi simulation identifies both isolated halos (distinct halos) and satellite halos (subhalos) embedded in larger halos and characterizes their properties separately. For any mass range the halo population is dominated by distinct halos (from more than 70 percent to 100 percent) but we include subhalos for accuracy. The Bolshoi simulation provides the distribution of halos in maximum circular velocity. The maximum circular velocity is found to be tightly correlated with the virial mass $M_{\mathrm{vir}}$ that is defined to be the mass bounded by the virial radius within which the mean density is equal to the virial overdensity $\Delta_{\text {vir }}$ times the mean cosmic density $\rho_{\mathrm{m}}=\Omega_{\mathrm{m}} \rho_{\text {crit }}$ ( $\rho_{\text {crit }}$ being the critical density of the Universe). For the above flat $\Lambda \mathrm{CDM}$ cosmological model $\Delta_{\text {vir }} \approx 360$ and the virial radius $r_{\text {vir }}$ is related to $M_{\text {vir }}$ by

$$
r_{\mathrm{vir}}=206.9 h^{-1}\left(\frac{M_{\mathrm{vir}}}{10^{12} h^{-1} M_{\odot}}\right)^{1 / 3} \mathrm{kpc}
$$

at redshift $z=0$ [52]. The halo mass function then follows from the combination of distinct halos and subhalos at fixed $M_{\text {vir }}$. The Bolshoi simulation does not sample halos beyond $M_{\mathrm{vir}} \approx(1-2) \times 10^{15} h^{-1} \mathrm{M}_{\odot}$. However, an analytic extrapolation of the halo mass function beyond the mass limit agrees with a larger volume simulation ( 'MultiDark') results [53] based on the same cosmological parameters.

\section{Statistically matching galaxies with halos: a bivariate log-normal distri- bution of stellar mass and velocity dispersion as a function of halo mass for early-type systems}

Abundance matching of a galaxy parameter (usually luminosity or stellar mass) with halo mass has been widely used recently $[15,45-50]$. For the early-type galaxy population we carry out a rigorous abundance matching of two parameters (stellar mass and velocity dispersion) of a galaxy with its host halo mass. There will result a bivariate distribution of stellar mass and velocity dispersion as a function of halo virial mass. The halo virial mass includes all mass within the halo, i.e. $M_{\mathrm{vir}}=M_{\mathrm{dm}}+M_{\star}$ where $M_{\mathrm{dm}}$ is the dark matter mass and $M_{\star}$ is the stellar mass of the galaxy. ${ }^{1}$ The radial dark matter density profile within the halo with $M_{\mathrm{dm}}$ is the unknown that we want to solve for. The galaxy is characterized primarily by its stellar mass $M_{\star}$ and velocity dispersion $\sigma$. The radial stellar mass density profile within the galaxy with $M_{\star}$ is modeled by a deprojected form of the Sérsic mass profile [54]. The effective radius $R_{\mathrm{e}}$ and the Sérsic index $n$ can be assigned according to the observed correlations with $M_{\star}$ and $\sigma$ (appendix A). Thus, the primary task is to assign simultaneously $M_{\star}$ and $\sigma$ to $M_{\text {vir }}$ for all halos in a statistically consistent manner.

The successful match between galaxies and halos (i.e. the assignment of $M_{\star}$ and $\sigma$ to $M_{\text {vir }}$ ) requires that the results satisfy all known statistical functions and correlations. These include the halo mass function, the galaxy stellar mass function, the galaxy velocity dispersion

\footnotetext{
${ }^{1}$ We ignore the mass of interstellar gas that is non-negligible for spiral systems because our work is concerned only with spheroidal systems. Black holes harboring at the galactic centers are also negligible for our analysis although we include them for completeness.
} 
function and the observed correlations between $M_{\text {vir }}$ and $M_{\star}$ and between $M_{\star}$ and $\sigma{ }^{2}$ The simultaneous assignment of $M_{\star}$ and $\sigma$ to $M_{\text {vir }}$ can be done through a bivariate probability distribution of $M_{\star}$ and $\sigma$ as a function of $M_{\text {vir }}$. The observed distribution of $\sigma$ at fixed $M_{\star}$ (or vice versa) is well described by a log-normal distribution for the early-type galaxy population $[41,55]$. The observed distribution of $M_{\star}$ at fixed $M_{\text {vir }}$ can also be described by a log-normal distribution [56]. These observations justify a choice of a bivariate normal distribution for the logarithmic values of $M_{\star}$ and $\sigma$ at fixed $M_{\text {vir }}$. Note that a bivariate normal distribution is not an accurate model for the total galaxy population, which includes both spheroidal and disk galaxies, because the observed distribution of $\sigma$ at fixed $M_{\star}$ for all galaxies is not log-normal but asymmetric.

We derive the bivariate normal distribution for the logarithmic values of $M_{\star}$ and $\sigma$ as a function of $M_{\mathrm{vir}}$ for the early-type galaxy population using an iterative procedure described in detail below. The parameters of the bivariate normal distribution are the mean $M_{\mathrm{vir}}-M_{\star}$ relation, the mean $M_{\mathrm{vir}}-\sigma$ relation, the standard deviations of $\log _{10}\left(M_{\star}\right)$ and $\log _{10}(\sigma)$, and the correlation coefficient between $\log _{10}\left(M_{\star}\right)$ and $\log _{10}(\sigma)$ as functions of $M_{\mathrm{vir}}$. The mean relations are based on an abundance-matching relation between $M_{\text {vir }}$ and $M_{\star}$ and the SDSS observed relation between $M_{\star}$ and $\sigma$. Starting with initial guesses of the standard deviations and the correlation coefficient we iterate keeping the mean relations fixed until the bivariate distribution reproduces the SDSS galaxy stellar mass and velocity dispersion functions and the $M_{\star}-\sigma$ relation up to the observational uncertainties.

\subsection{The detailed procedure}

We describe in detail the procedure ${ }^{3}$ of generating a bivariate log-normal distribution of stellar mass $\left(M_{\star}\right)$ and stellar velocity dispersion $(\sigma)$ as a function of halo virial mass $\left(M_{\mathrm{vir}}\right)$, or a bivariate normal distribution of $Y \equiv \log _{10}\left(M_{\star} / M_{\odot}\right)$ and $Z \equiv \log _{10}\left(\sigma / \mathrm{km} \mathrm{s}^{-1}\right)$ as a function of $X \equiv \log _{10}\left(M_{\text {vir }} / M_{\odot}\right)$. We use a sufficiently large comoving volume of $4 \times 10^{9} \mathrm{Mpc}^{3}$ to keep high precision in statistical match toward the large mass limit. Our results are then limited only by the accuracies of the input statistical quantities. Throughout a system refers to the combination of a halo (whether it is isolated/distinct or a subhalo embedded in a larger halo) and the central galaxy embedded at the center of the halo.

The statistical quantities of our use for galaxies are (1) the stellar mass function (SMF) for all-type galaxies, (2) the SMF and the velocity dispersion function (VDF) for early-type galaxies, (3) the number fraction of early-type galaxies $f_{E}$ as a function of $M_{\star}$, and (4) the distribution of early-type galaxies in the stellar mass-velocity dispersion plane, to be referred to as the $M_{\star}-\sigma$ relation. The $M_{\star}-\sigma$ relation for early-type galaxies with $C_{r}>2.86$ is derived and presented here while all the other have been published [41]. Stellar masses are based on the Chabrier initial mass function throughout. The statistical quantity of our use for halos is the halo mass function (HMF) that includes both distinct halos and subhalos. Throughout a statistical function (i.e. SMF, VDF, or HMF) is denoted by $\phi$ and expressed as comoving number density per unit logarithmic interval of the variable under consideration.

1. The first step is to find a mean relation between $M_{\star}$ and $M_{\text {vir }}$ for all-type galaxies from the SMF and the HMF using the abundance matching method [15, 45-50]. This method uses a prior knowledge (or assumption) of the intrinsic scatter of one variable at the other. Observational studies find $s_{Y}=0.16-0.17$ for the intrinsic scatter of $Y$ at fixed $X$ insensitive to the value of $X$ [56-58]. We adopt $s_{Y}=0.17$ [56]. We follow the procedure demonstrated in

\footnotetext{
${ }^{2}$ Note that no direct observational constraint is available for the correlation between $M_{\mathrm{vir}}$ and $\sigma$.

${ }^{3}$ The reader who is only interested in the result may skip this lengthy subsection.
} 

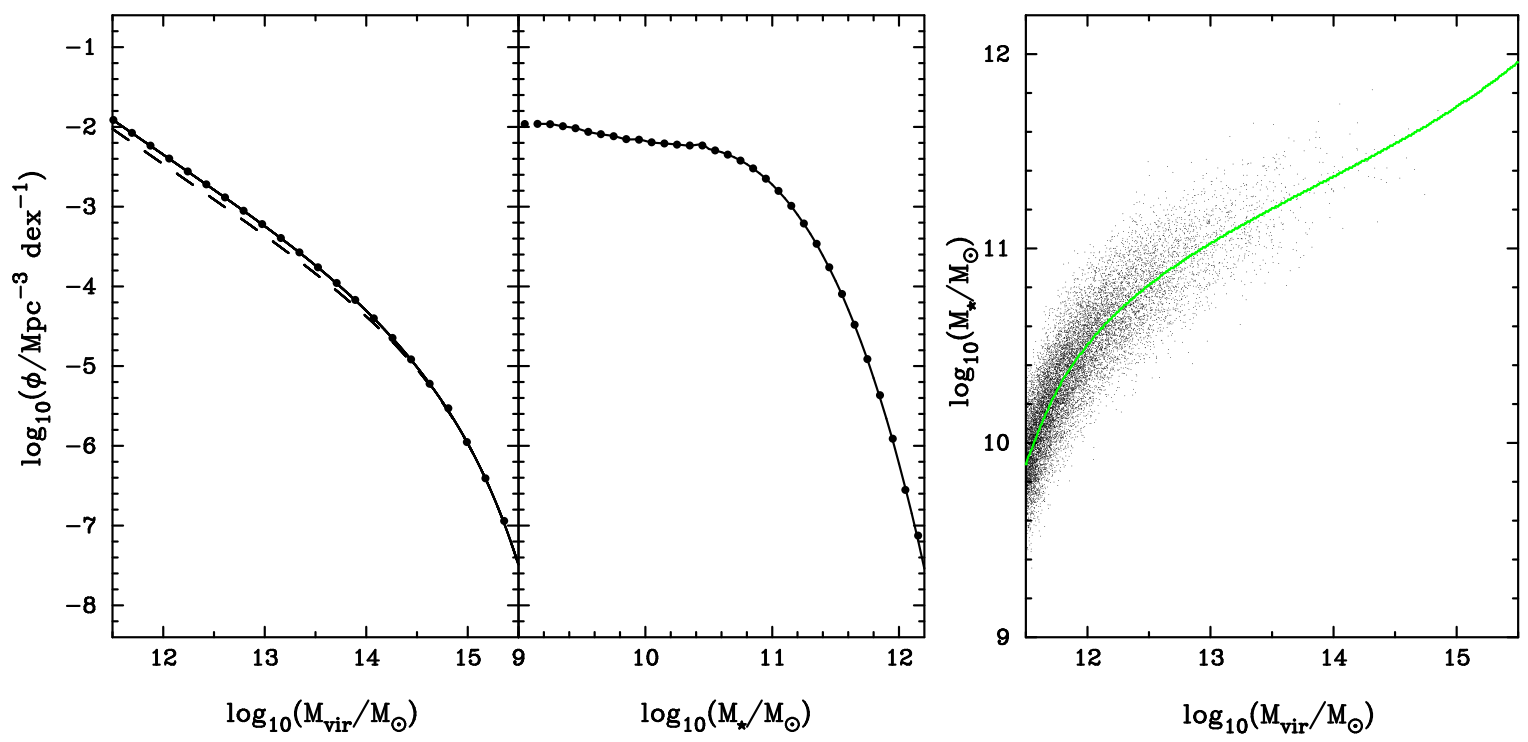

Figure 1. The first panel shows the halo mass function from the Bolshoi N-body simulation. The solid curve includes all halos (i.e. both distinct and subhalos) while the dashed curve includes only distinct halos. Data points show mock halos within a comoving volume of $4 \times 10^{9} \mathrm{Mpc}^{3}$. The second panel shows the SDSS galaxy stellar mass function for all-type galaxies. The curve is the SDSS measured function while data points are from galaxies assigned to the halos according to the relation shown in the last panel. The last panel shows the final abundance matching $M_{\mathrm{vir}}-M_{\star}$ relation with a Gaussian intrinsic (constant) scatter of 0.17 for $\log \left(M_{\star}\right)$ at fixed $M_{\text {vir }}$.

[50]. Initially we obtain an approximate abundance matching relation assuming zero intrinsic scatter. This initial relation is biased at large $X$ owing to the ignored scatter. The difference between the initial relation and the unknown true relation is to be referred to as bias. The remaining task is to estimate the bias and thus the true mean relation as well. We do this iteratively using a Monte-Carlo method in the following way. Let us first consider the initial mean relation as a surrogate of the true mean relation. We generate halos from the HMF and then assign $Y$ to $X$ through the surrogate mean $X-Y$ relation and the intrinsic scatter $s_{Y}=0.17$ assuming the Gaussian model. From these mock systems we derive a mock SMF which is of course quite different from the observed SMF because of the biased input surrogate relation. Now obtain a mock abundance matching relation between this mock SMF and the HMF ignoring the intrinsic scatter. Then the difference between this mock relation and the input surrogate relation is our first estimate of the bias. Using this bias we correct the initial abundance matching relation between the observe SMF and the HMF. With the corrected relation we do the simulation all over again. There results a better corrected relation. We iterate this simulation until the mock SMF matches the observed SMF. Usually a few iterations suffice. Fig. 1 shows the reproduced HMF and SMF and the final $X-Y$ plane. The total number of the generated systems with $M_{\text {vir }} \geq 10^{10.5} \mathrm{M}_{\odot}$ is $\simeq 1.866 \times 10^{8}$.

2. We use the above $X-Y$ plane to convert the observed early-type fraction of systems as a function of $Y$ (stellar mass) to that at fixed $X$ (halo mass). Let $f_{\mathrm{E}}(X, Y)$ be the early-type fraction as a function of $X$ and $Y$. If $f_{\mathrm{E}}(X, Y)$ were known, $f_{\mathrm{E}}(X)$, the fraction at $X$ would be simply $\int f_{\mathrm{E}}(X, Y) P(Y \mid X) d Y$ where $P(Y \mid X)$ is the probability distribution of $Y$ at $X$ 

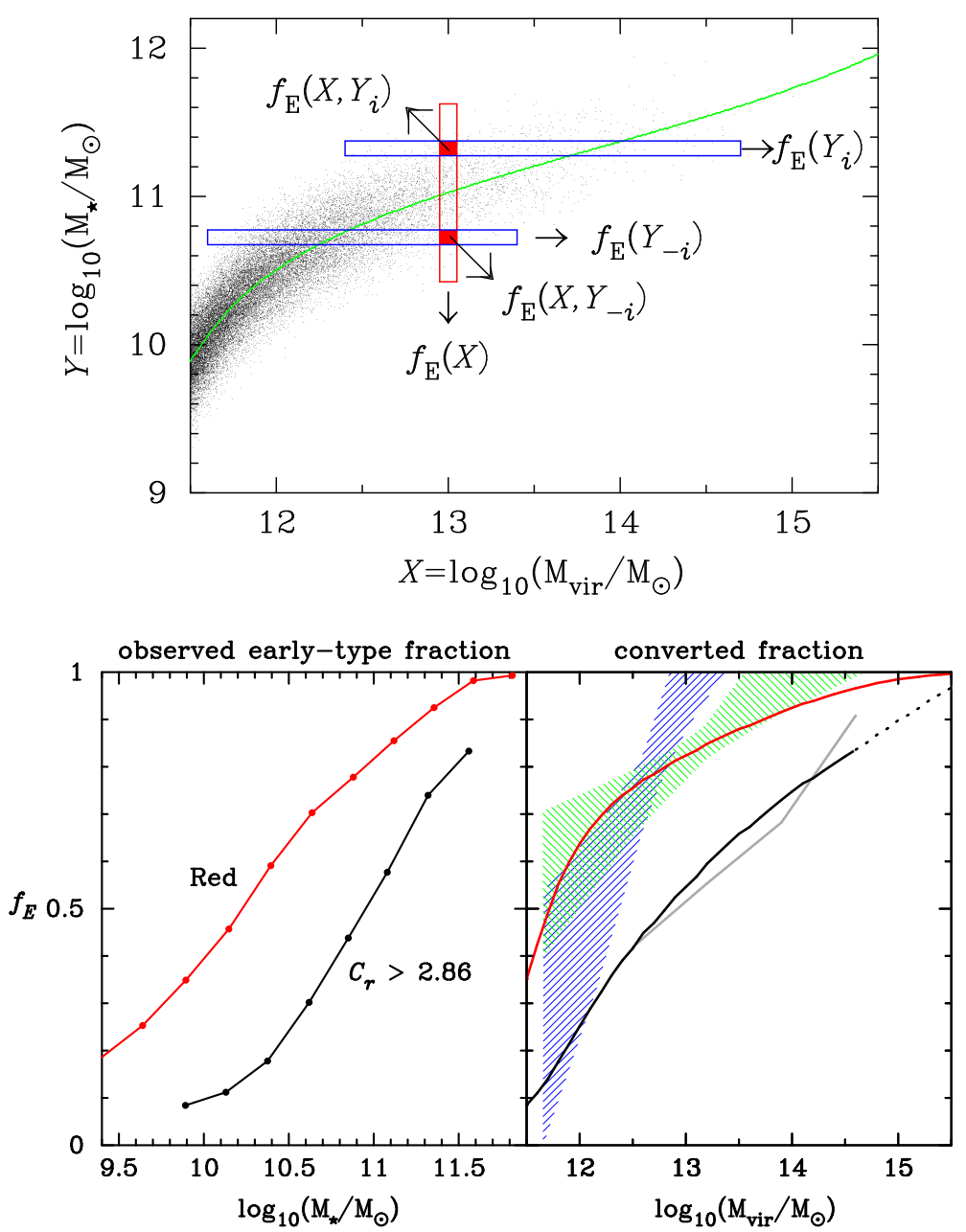

Figure 2. (Top) A schematic view of converting the early-type fraction as a function of $Y$ to that at fixed $X$. We use $f_{\mathrm{E}}(X)=\sum_{i}\left[f_{\mathrm{E}}\left(X, Y_{i}\right) w_{i}+f_{\mathrm{E}}\left(X, Y_{-i}\right) w_{-i}\right] \approx \sum_{i}\left[f_{\mathrm{E}}\left(Y_{i}\right)+f_{\mathrm{E}}\left(Y_{-i}\right)\right] w_{i}$ where $w_{i}$ and $w_{-i}$ are statistical weights satisfying $\sum_{i}\left(w_{i}+w_{-i}\right)=1$. The last approximation follows from $w_{-i}=w_{i}$ and the assumption that $\left\{\left[f_{\mathrm{E}}\left(X, Y_{i}\right)-f_{\mathrm{E}}\left(Y_{i}\right)\right]+\left[f_{\mathrm{E}}\left(X, Y_{-i}\right)-f_{\mathrm{E}}\left(Y_{-i}\right)\right]\right\} w_{i} \approx 0$, which is plausible (see the texts). (Bottom) The left panel shows the early-type number fraction (black curve) of SDSS galaxies as a function of stellar mass. We use the observed fraction of galaxies with $C_{r}>2.86$ for $10^{9.9} M_{\odot} \leq M_{\star} \leq 10^{11.6} M_{\odot}$ since outside this range the measured fraction is less reliable [41]. The red galaxy number fraction [41] is also shown for comparison. The right panel shows the early-type number fraction (black solid curve) as a function of halo mass $\left(M_{\mathrm{vir}}\right)$ converted from the observed fraction at $M_{\star}$ using the $M_{\mathrm{vir}}-M_{\star}$ plane shown above. The dotted curve is a linear extrapolation as a function of $\log _{10}\left(M_{\mathrm{vir}}\right)$ for the high-mass part $\left(M_{\mathrm{vir}}>10^{14.5} M_{\odot}\right)$. The gray curve is the number fraction of visually selected early-type galaxies based on halo occupation statistics results [42]. The red curve shows the converted red number fraction for our red galaxies. The hatched regions show observational results (95\% confidence regions) on the red fraction based on SDSS data and satellite kinematics (green/blue region based on the observed fraction as a function of luminosity/stellar mass) [56]. There are consistencies for both early-type fractions and red fractions. 
that comes from the $X-Y$ relation. In our case $P(Y \mid X)$ is the Gaussian distribution with a standard deviation of $s_{Y}=0.17$. Without $f_{\mathrm{E}}(X, Y)$ we use the following approximation

$$
f_{\mathrm{E}}(X) \approx \int f_{\mathrm{E}}(Y) P(Y \mid X) d Y
$$

where $f_{\mathrm{E}}(Y)$ is the observed fraction at $Y$. This approximation is obtained as follows:

$$
\begin{aligned}
f_{\mathrm{E}}(X) & =\int f_{\mathrm{E}}(X, Y) P(Y \mid X) d Y \\
& \rightarrow \sum_{i}\left[f_{\mathrm{E}}\left(X, Y_{i}\right) P\left(Y_{i} \mid X\right)+f_{\mathrm{E}}\left(X, Y_{-i}\right) P\left(Y_{-i} \mid X\right)\right] \Delta Y \\
& =\sum_{i}\left[f_{\mathrm{E}}\left(X, Y_{i}\right) w_{i}+f_{\mathrm{E}}\left(X, Y_{-i}\right) w_{-i}\right],
\end{aligned}
$$

where $Y_{i}(i=1,2,3, \cdots)$ are greater than the mean $\langle Y\rangle, Y_{-i}=2\langle Y\rangle-Y_{i}$ (see the top panel of Fig. 2), and statistical weights $w_{i}\left[\equiv P\left(Y_{i} \mid X\right) \Delta Y\right]$ and $w_{-i}\left[\equiv P\left(Y_{-i} \mid X\right) \Delta Y\right]$ satisfy $\sum_{i}\left(w_{i}+w_{-i}\right)=1$ and $w_{-i}=w_{i}$ as well for the symmetric Gaussian distribution. It then follows

$$
\begin{aligned}
f_{\mathrm{E}}(X) & =\sum_{i}\left[f_{\mathrm{E}}\left(X, Y_{i}\right)+f_{\mathrm{E}}\left(X, Y_{-i}\right)\right] w_{i} \\
& =\sum_{i}\left[f_{\mathrm{E}}\left(Y_{i}\right)+f_{\mathrm{E}}\left(Y_{-i}\right)\right] w_{i}+\sum_{i}\left[\delta f_{\mathrm{E}}\left(X, Y_{i}\right)+\delta f_{\mathrm{E}}\left(X, Y_{-i}\right)\right] w_{i},
\end{aligned}
$$

where $f_{\mathrm{E}}(Y)=\int f(X, Y) P(X \mid Y) d X[P(X \mid Y)$ being the probability distribution of $X$ at fixed $Y]$ and $\delta f_{\mathrm{E}}(X, Y) \equiv f_{\mathrm{E}}(X, Y)-f_{\mathrm{E}}(Y)$. But, we expect $\delta f_{\mathrm{E}}\left(X, Y_{i}\right) \approx-\delta f_{\mathrm{E}}\left(X, Y_{-i}\right)$ for $Y_{i}$ close to $\langle Y\rangle$ (i.e. small values of $i$ ) assuming that $f_{\mathrm{E}}(X, Y)$ is a smooth distribution and the shape of $P(X \mid Y)$ is slowly varying with $Y$. For large values of $i$ the approximation $\delta f_{\mathrm{E}}\left(X, Y_{i}\right) \approx-\delta f_{\mathrm{E}}\left(X, Y_{-i}\right)$ may not be good enough but $w_{i} \approx 0$ for the Gaussian distribution of $Y$. Hence, we have $\sum_{i}\left[\delta f_{\mathrm{E}}\left(X, Y_{i}\right)+\delta f_{\mathrm{E}}\left(X, Y_{-i}\right)\right] w_{i} \approx 0$ and equation (3.3) is reduced to equation (3.1).

Fig. 2 shows the functional behaviors of observed and converted fractions. The earlytype $\left(C_{r}>2.86\right)$ fraction is compared with the red fraction. At a given stellar mass the red fraction is significantly higher than the early-type fraction. This implies that significant fractions of red galaxies do not possess concentrated light distributions. The converted fractions as functions of $M_{\text {vir }}$ are compared with recent independent results. Notice that our early-type fraction based on luminosity concentration index is in good agreement with a fraction based on visual inspection of galaxy morphology [42]. Our red fraction is also consistent with independent results based on color selection [56]. We obtain the HMF for early-type systems by multiplying the number density for all-type halos by $f_{\mathrm{E}}(X)$. Fig. 3 shows the HMF for early-type systems.

3. Assign $Y$ to $X$ for the early-type population using the same procedure of step 1 based on the early-type HMF from step 2 and the observed early-type SMF. Fig. 4 shows the resulting HMF and SMF and the $X-Y$ plane for the early-type population. We obtain $\simeq 6.9 \times 10^{6}$ early-type systems with $X$ and $Y$ assigned.

4. For the early-type systems from step 3, we assign $Z$ to $Y$ using only the observed $Y-Z$ relation (Fig. 5) ignoring the correlation between $X$ and $Z$. In this way we obtain an approximate set $\{X, Y, Z\}$. This set is approximate in the sense that parameter correlations 


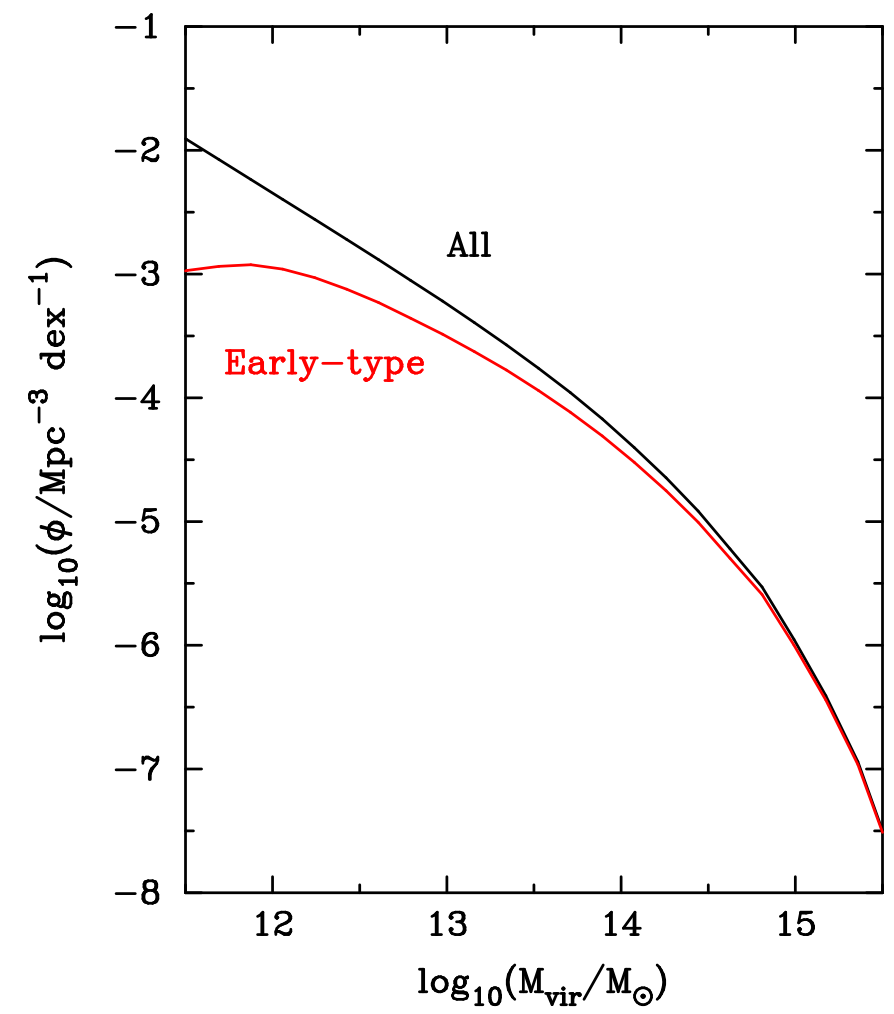

Figure 3. Halo mass function for those halos embedding early-type galaxies at their centers (red curve) derived by multiplying the total halo mass function (black curve) by the early-type fraction (shown in Fig. 2) as a function of $M_{\mathrm{vir}}$.

and scatters have not yet been determined. Nevertheless the mean relations among three parameters should be accurate as long as the distributions in the two-parameter planes are assumed symmetric [observations support the symmetric distributions of $Y$ (at fixed $X$ ) and $Z$ (at fixed $Y$ ) for the early-type population]. This set allows us to obtain a crude estimate of $s_{Z}$ (standard deviation of $Z$ ) at fixed $X$.

5. Let us now assume that $Y$ and $Z$ follow a bivariate normal distribution at fixed $X$. The probability density function is given by

$$
P(Y, Z \mid X)=\frac{1}{2 \pi s_{Y} s_{Z} \sqrt{1-\rho_{Y Z}^{2}}} \exp \left[-\frac{1}{2\left(1-\rho_{Y Z}^{2}\right)} g(Y, Z)\right]
$$

with

$$
g(Y, Z)=\frac{\left(Y-\mu_{Y}\right)^{2}}{s_{Y}^{2}}+\frac{\left(Z-\mu_{Z}\right)^{2}}{s_{Z}^{2}}-\frac{2 \rho_{Y Z}\left(Y-\mu_{Y}\right)\left(Z-\mu_{Z}\right)}{s_{Y} s_{Z}},
$$

where $\mu_{Y}$ and $\mu_{Z}$ are the mean values and $\rho_{Y Z}$ is the correlation coefficient at fixed $X$. The values of $\mu_{Y}$ and $\mu_{Z}$ come from step 4. We assume $s_{Y}=0.17$ [56] independent of $X$. Parameters $s_{Z}$ and $\rho_{Y Z}$ are the unknowns to be determined. With the first guess of $s_{Z}$ from step 4 and an initial guess of $\rho_{Y Z}$ (e.g. 0.4) we generate our first mock set including the relevant correlation. This mock set reproduces the SMF because we are using the bias corrected abundance matching $X-Y$ relation. However, the set does not reproduce 

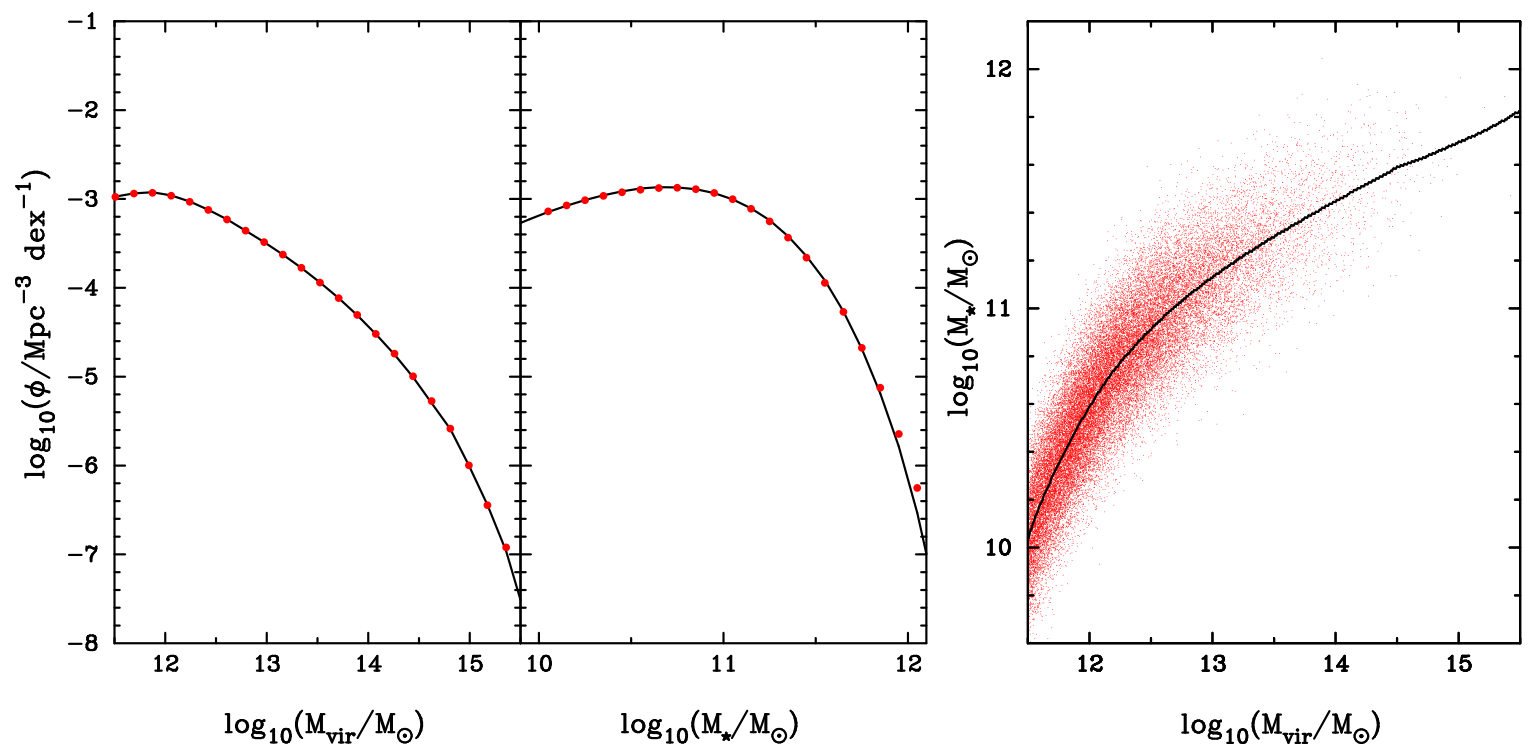

Figure 4. Same as Fig. 1 but for early-type galaxy-halo systems.
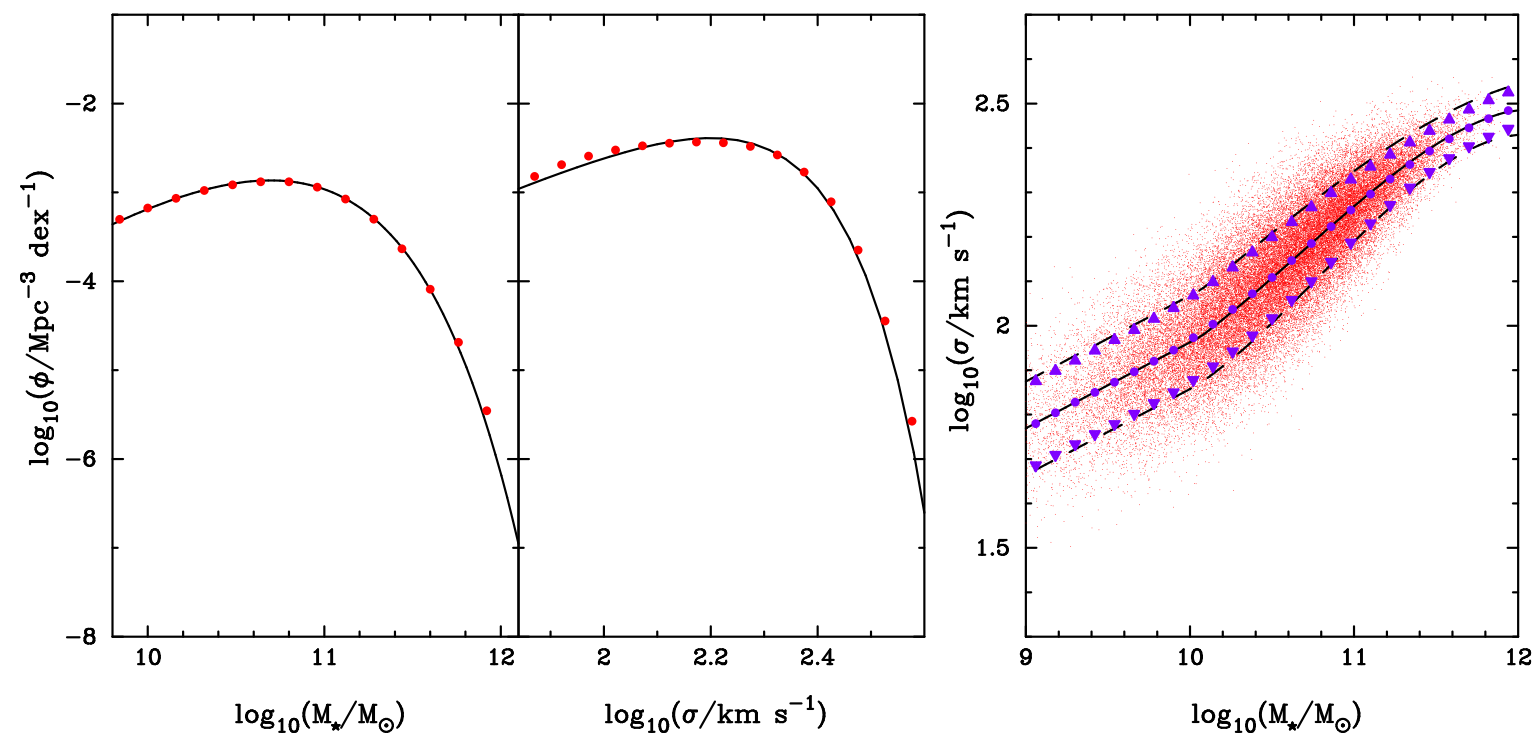

Figure 5. (Left) The black curves are the SDSS stellar mass $\left(M_{\star}\right)$ and stellar velocity dispersion $(\sigma)$ functions for early-type galaxies with $C_{r}>2.86$ [41]. Red points are derived from the data points shown in the right panel. (Right) The black full and dashed curves represent our measured median values and standard deviations for SDSS early-type galaxies based on the data analyzed in [41]. Red data points show a sample of mock galaxies produced from our final bivariate distribution of $M_{\star}$ and $\sigma$ as a function of $M_{\text {vir }}$ to be shown in section 3.2. The purple filled circles and upright/upside-down triangles are respectively the median values and standard deviations of the data points. 
the observed VDF and the observed stellar mass-velocity dispersion relation because of the inaccurate $s_{Z}$ and the unknown $\rho_{Y Z}$.

6. We use the mock set itself to estimate the bias in $s_{Z}$. This is done in the following way. First, calculate the standard deviation of $Z$ at fixed $Y, s_{Z}(Y)$. Next, take the lower dimensional set $\{X, Y\}$ from the mock set and then assign $Z$ to $Y$ using $s_{Z}(Y)$ just calculated, pretending the correlation in the mock set were unknown. Thus, we get a biased set $\{X, Y, Z\}$. Finally, we calculate $s_{Z}$ at fixed $X$ from this biased set and then compare it with that of the mock set to estimate the bias.

7. Correct $s_{Z}$ for the bias estimated in step 6 . Using this corrected $s_{Z}$ generate a revised mock set $\{X, Y, Z\}$ using the bivariate normal probability distribution of $Y$ and $Z$ at fixed $X$. We can check that this revised mock set results in an improved match to the observed VDF. We note that the resulting VDF has much to do with $s_{Z}$ but little to do with $\rho_{Y Z}$. The correlation $\rho_{Y Z}$ has much to do with the resulting $Y-Z$ relation, its mean relation and the dispersion of $Z$ at fixed $Y$. Hence, for the given $s_{Z}(X)$ we adjust $\rho_{Y Z}$ so that the resulting $Y-Z$ relation matches the observed stellar mass-velocity dispersion as closely as possible. For the sake of simplicity we allow $\rho_{Y Z}$ to be only a linear function of $X$ with broken slopes.

8. If the results of step 7 are not satisfactory go back to step 6 and iterate. Initially we focus on reproducing the VDF by correcting $s_{Z}(X)$ with a constant $\rho_{Y Z}$. Eventually the initially adopted $\rho_{Y Z}$ will be modified as the observed median relation between stellar mass and velocity dispersion cannot be well reproduced with a constant $\rho_{Y Z}$. We find that with a constant $\rho_{Y Z}=0.35$ the resulting VDF matches well the observed VDF for $\sigma>100 \mathrm{~km} \mathrm{~s}^{-1}$. This means that through the iteration we have found a proper functional behavior of $s_{Z}(X)$ (see Fig. 6). On the other hand, the resulting mean value of $Z$ at $Y$ deviates downward from the observed relation towards the high mass end. This requires us to correct the constant $\rho_{Y Z}$. After trial and error we find that a varying $\rho_{Y Z}(X)$ with enhanced values for relatively more massive systems, as shown in Fig. 6, can reproduce the observed median relation between stellar mass and velocity dispersion (see Fig. 5). However, for this model of $\rho_{Y Z}(X)$ the intrinsic scatter of $Z$ at fixed $Y$ is slightly underestimated as can be seen in Fig. 5. This lowered dispersion is a consequence of the strengthened correlation. This manifests the difficulty of matching the VDF and the stellar mass-velocity dispersion relation simultaneously to a high precision. Some compromise is necessary and we have chosen to give more weight to the VDF for $\sigma>100 \mathrm{~km} \mathrm{~s}^{-1}$ and the median stellar mass-velocity relation but less weight to the the intrinsic scatter of velocity dispersion at fixed stellar mass as the observational error of the latter has not been quantified.

\subsection{The result: a semi-empirical catalog of early-type galaxy-halo systems with some parameters undetermined}

We obtain a final set $\left\{M_{\text {vir }}, M_{\star}, \sigma\right\}$ of $\simeq 6.9 \times 10^{6}$ mock early-type systems with $M_{\text {vir }}>$ $10^{10.5} M_{\odot}$ for a training comoving volume of $4 \times 10^{9} \mathrm{Mpc}^{3}$. A subset of 50,000 systems is displayed in Fig. 7 . The projected $M_{\text {vir }}-M_{\star}$ relation is compared with recent available observational results based on other methods including galaxy-galaxy weak lensing [59], halo occupation statistics [42], and kinematics of satellite galaxies [56]. Our mean $M_{\mathrm{vir}}-M_{\star}$ relation lies near the median of other results for $M_{\text {vir }}<10^{13} M_{\odot}$ but lies lower down to -0.2 dex for $M_{\text {vir }}>10^{13} M_{\odot}$. We note that this high mass part behavior of the abundance matching $M_{\text {vir- }}$ $M_{\star}$ relation in comparison to other methods is also found for all-type galaxies [49]. However, it turns out that this difference has relatively minor effect on our results on dark matter 

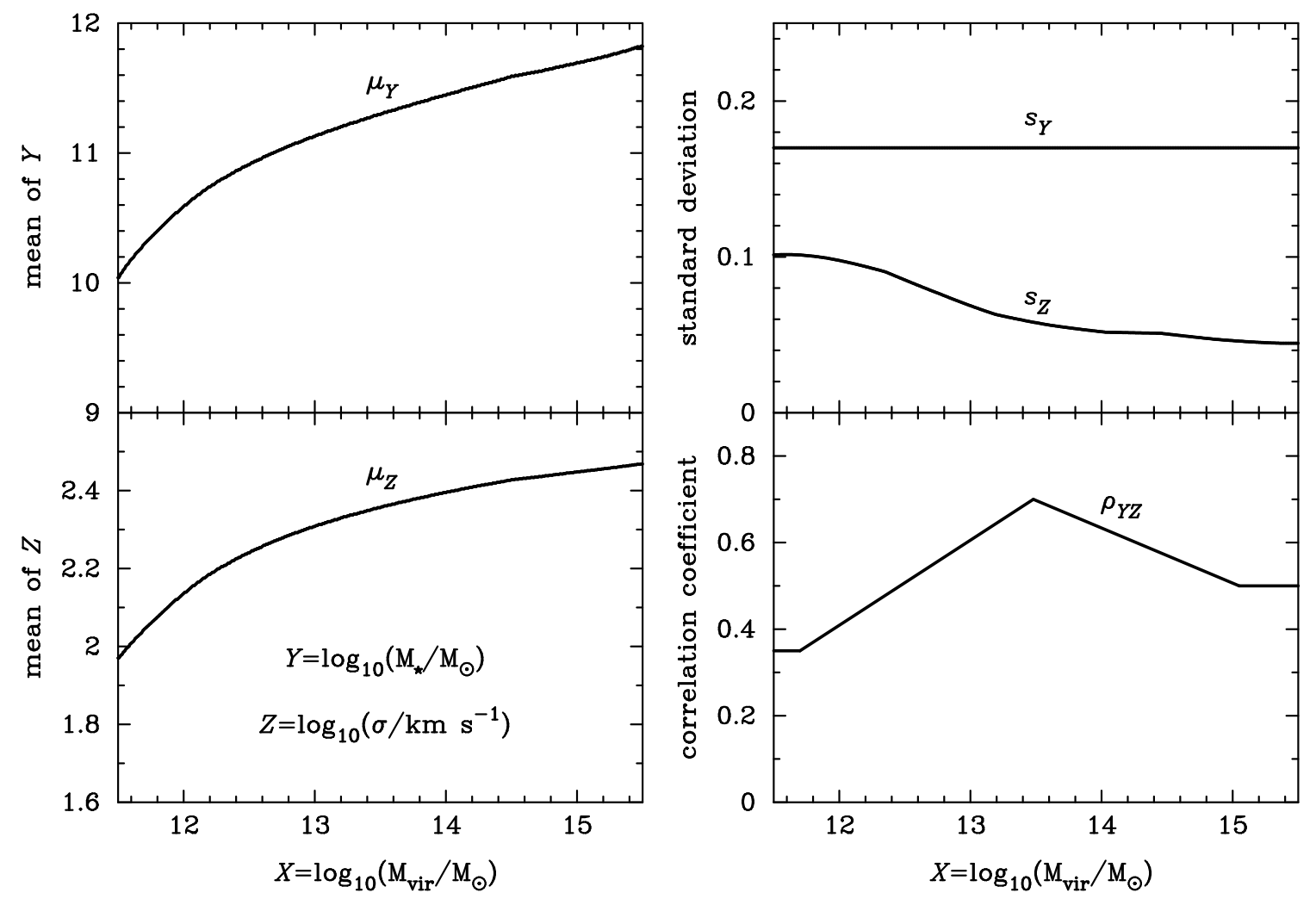

Figure 6. Parameters of the bivariate normal distribution of $Y\left[\equiv \log _{10}\left(M_{\star} / M_{\odot}\right)\right]$ and $Z[\equiv$ $\left.\log _{10}\left(\sigma / \mathrm{km} \mathrm{s}^{-1}\right)\right]$ as functions of $X\left[\equiv \log _{10}\left(M_{\text {vir }} / M_{\odot}\right)\right]$.

density profiles. The $M_{\mathrm{vir}}-\sigma$ relation for early-type galaxies is derived here for the first time. Abundance matching $M_{\mathrm{vir}}-\sigma$ relations for all-type galaxies can be found in [50,60].

We have essentially constructed a realistic catalog of early-type systems by simultaneously assigning stellar mass $\left(M_{\star}\right)$ and stellar velocity dispersion $(\sigma)$ to halo virial mass $\left(M_{\text {vir }}\right)$ in the way that the empirical $M_{\star}-\sigma$ and $M_{\text {vir }}-M_{\star}$ relations are preserved. This catalog is not fully empirical because $M_{\text {vir }}$ is drawn from dissipationless N-body simulations rather than observations. We refer to this catalog as a semi-empirical catalog (SEC). We emphasize that the dissipationless N-body prediction of halo mass function is robust under the $\Lambda$ CDM paradigm as it is based only on gravitational physics. Abundance matching of the halo mass function with galaxy properties such as stellar mass has already produced meaningful results in the literature including the average relation between $M_{\star}$ and $M_{\text {vir }}$ and its evolution with redshift and the clustering of galaxies at different redshifts [15, 45, 47-49]. The galaxy with $M_{\star}$ and $\sigma$ assigned can be further assigned its stellar mass density profile through their observed correlations with the effective radius $R_{\mathrm{e}}$ and the Sérsic index $n$. These correlations are described in appendix A. For this SEC, however, dark matter density profiles are missing. We turn next to dynamical modeling of the systems in the SEC. 

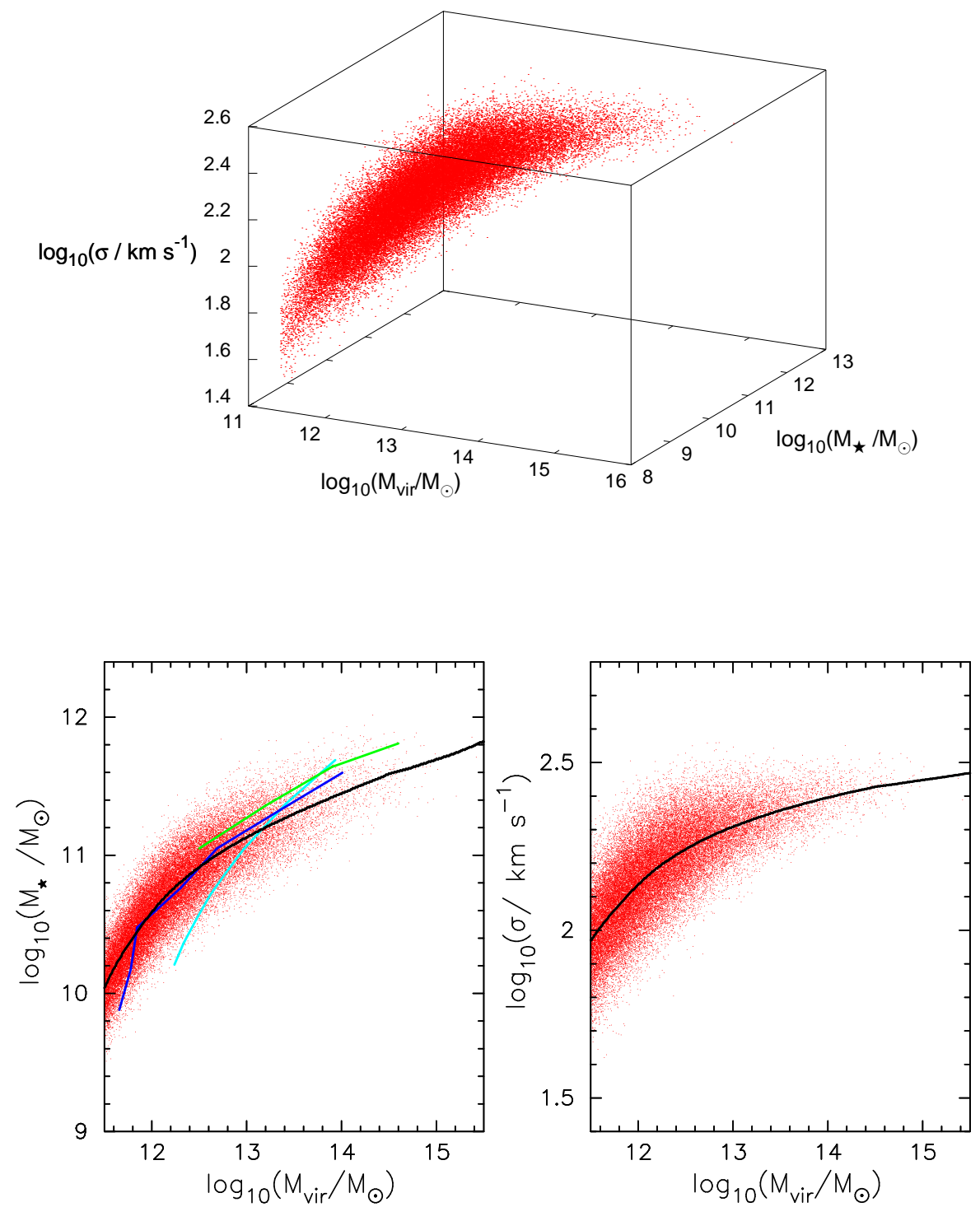

Figure 7. (Top) The bivariate normal distribution of $\log _{10}\left(M_{\star} / M_{\odot}\right)$ and $\log _{10}\left(\sigma / \mathrm{km} \mathrm{s}^{-1}\right)$ as a function of $\log _{10}\left(M_{\mathrm{vir}} / M_{\odot}\right)$ for early-type galaxy-halo systems where $M_{\mathrm{vir}}$ is the virial mass of the halo embedding an early-type galaxy at the center and $M_{\star}$ and $\sigma$ are the stellar mass and central stellar velocity dispersion of the galaxy respectively. (Bottom) Two projections of the three-dimensional distribution. Black curves are the mean relations. The mean $M_{\mathrm{vir}}-M_{\star}$ relation is compared with recent observational results on early-type or red galaxies based on galaxy-galaxy weak lensing (blue curve) [59], halo occupation statistics (green) [42] and satellite kinematics (cyan) [56].

\section{Jeans dynamical modeling of early-type galaxy-halo systems}

\subsection{The spherical Jeans equation}

For the early-type systems in the SEC described in section 3.2 we can perform Jeans dynamical modeling to constrain the dark matter density profile of the embedding halo because the stellar velocity dispersion depends not only on the stellar mass distribution but also on the 
dark matter distribution. We focus on the spherical Jeans equation [61] given by

$$
\frac{d\left[\rho_{\star}(r) \sigma_{\mathrm{r}}^{2}(r)\right]}{d r}+2 \frac{\beta(r)}{r}\left[\rho_{\star}(r) \sigma_{\mathrm{r}}^{2}(r)\right]=-G \frac{\rho_{\star}(r) M(r)}{r^{2}},
$$

where $\sigma_{\mathrm{r}}(r)$ is the radial stellar velocity dispersion at radius $r, \rho_{\star}(r)$ is the three-dimensional stellar mass density at $r, M(r)$ is the total (i.e. stellar plus dark) mass enclosed within $r$, i.e. $M(r)=M_{\star}(r)+M_{\mathrm{dm}}(r)$, and $\beta(r)$ is the velocity dispersion anisotropy at $r$ given by

$$
\beta(r)=1-\frac{\sigma_{\theta}^{2}(r)+\sigma_{\phi}^{2}(r)}{2 \sigma_{\mathrm{r}}^{2}(r)},
$$

where $\sigma_{\theta}(r)$ and $\sigma_{\phi}(r)$ are the tangential velocity dispersions in spherical coordinates. An integral solution of the Jeans equation for $\sigma_{\mathrm{r}}(r)$ is given in appendix B.

The LOSVD of stars at projected radius $R$ on the sky $\sigma_{\text {los }}(R)$ is given by

$$
\sigma_{\mathrm{los}}^{2}(R)=\frac{1}{\Sigma_{\star}(R)} \int_{R^{2}}^{\infty} \rho_{\star}(r) \sigma_{\mathrm{r}}^{2}(r)\left[1-\frac{R^{2}}{r^{2}} \beta(r)\right] \frac{d r^{2}}{\sqrt{r^{2}-R^{2}}},
$$

where $\Sigma_{\star}(R)$ is the two-dimensional stellar mass density projected on the sky. The stellar mass weighted $j$-th power of LOSVD within an aperture of radius $R$ is then given by

$$
\left\langle\sigma_{\text {los }}^{j}\right\rangle(R)=\frac{\int_{0}^{R} \Sigma_{\star}\left(R^{\prime}\right) \sigma_{\text {los }}^{j}\left(R^{\prime}\right) R^{\prime} d R^{\prime}}{\int_{0}^{R} \Sigma_{\star}\left(R^{\prime}\right) R^{\prime} d R^{\prime}} .
$$

Finally, assuming that stellar mass follows luminosity the quantity to match the SDSS velocity dispersion $\sigma$ is given by

$$
\sigma=\left\langle\sigma_{\text {los }}\right\rangle\left(R=R_{\mathrm{e}} / 8\right) .
$$

\subsection{Models for the dark matter distribution}

Initially all mass is supposed to follow a density profile similar to the NFW profile [62-64], as predicted by N-body simulations. As the galaxy is formed and settled at the center of the halo, the separate stellar mass distribution is embedded in the remaining dark matter distribution. While the stellar mass distribution can be represented by the Sérsic profile as indicated by observations, the remaining dark matter distribution is completely unknown.

With the assumption that the dark matter distribution has been smoothly readjusted from the initial NFW(-like) profile, we model the unknown dark matter distribution using a generalized NFW density profile, referred to as $\alpha \mathrm{NFW}$ throughout, given by

$$
\rho_{\alpha \mathrm{NFW}}(r)=\frac{\rho_{\mathrm{s}}}{\left(r / r_{\mathrm{s}}\right)^{\alpha}\left(1+r / r_{\mathrm{s}}\right)^{3-\alpha}},
$$

where $\alpha$ is the inner density power-law slope ( $\alpha=1$ being the NFW value), $r_{\mathrm{s}}$ is the scale radius related to the concentration parameter $c_{\mathrm{vir}}$ via

$$
c_{\mathrm{vir}}=\frac{r_{\mathrm{vir}}}{r_{\mathrm{s}}},
$$

and the parameter $\rho_{\mathrm{s}}$ is related to the dark matter mass within the virial radius $M_{\mathrm{dm}}(=$ $\left.M_{\text {vir }}-M_{\star}\right)$ via

$$
\rho_{\mathrm{s}}=\frac{M_{\mathrm{dm}}}{4 \pi r_{\mathrm{s}}^{3} f_{\alpha}\left(c_{\mathrm{vir}}\right)}
$$


where the function $f_{\alpha}(x)$ is given by

$$
f_{\alpha}(x)=\int_{0}^{x} \frac{t^{2-\alpha}}{(1+t)^{3-\alpha}} d t .
$$

We also consider the Einasto profile [65], that is suggested by relatively more recent dissipationless N-body simulations [63, 64], given by

$$
\rho_{\operatorname{Ein}}(r)=\rho_{-2} \exp \left\{-(2 / \tilde{\alpha})\left[\left(r / r_{-2}\right)^{\tilde{\alpha}}-1\right]\right\},
$$

where $r_{-2}$ is the radius at which the logarithmic slope of the density is -2 and from which we define another concentration parameter given by

$$
\tilde{c}_{\mathrm{vir}}=\frac{r_{\mathrm{vir}}}{r_{-2}}
$$

and the parameter $\rho_{-2}$ is related to the dark matter mass via

$$
\rho_{-2}=\frac{M_{\mathrm{dm}}}{4 \pi r_{-2}^{3} \tilde{f}_{\tilde{\alpha}}\left(\tilde{c}_{\mathrm{vir}}\right)},
$$

where the function $\tilde{f}_{\tilde{\alpha}}(x)$ is given by

$$
\tilde{f}_{\tilde{\alpha}}(x)=\frac{1}{\tilde{\alpha}}\left(\frac{2}{\tilde{\alpha}}\right)^{-3 / \tilde{\alpha}} \exp \left(\frac{2}{\tilde{\alpha}}\right) \gamma\left(\frac{3}{\tilde{\alpha}}, \frac{2}{\tilde{\alpha}} x^{\tilde{\alpha}}\right)
$$

where $\gamma(a, b)$ is the incomplete gamma function.

\subsection{Results on dark matter density profiles}

\subsubsection{Restricted results based only on the SDSS velocity dispersion}

For a system in the SEC with $M_{\text {vir }}$ and the other parameters assigned the dark matter distribution given by the $\alpha \mathrm{NFW}$ (or Einasto) profile is completely specified by two parameters $\alpha$ and $c_{\text {vir }}$ (or, $\tilde{\alpha}$ and $\tilde{c}_{\text {vir }}$ ). Without additional information the Jeans equation can be used only to solve for one of the two parameters $\alpha$ and $c_{\text {vir }}$ when the other and the velocity dispersion anisotropy $\beta(r)$ are specified in advance. This can be done by equating the theoretical velocity dispersion given by equation (4.5) to the SDSS velocity dispersion. We consider the case of constant anisotropy $\beta$ and assign its value using

$$
\beta=\left\{\begin{array}{cl}
x & \text { if } 0 \leq x<0.5 \\
1.8 x & \text { if }-0.5<x<0
\end{array},\right.
$$

where $x$ is an uniform random variable and we are using the prior $-0.9<\beta<0.5$ from stellar dynamics analyses of handfuls of early-type galaxies in the literature [6, 66, 67]. We further consider the case that either $\alpha=1$ or $c_{\text {vir }}$ is fixed at the Bolshoi predicted value for all halos (i.e. the weighted mean for distinct halos and subhalos). In some cases, for a given value of $\beta$ and the rest of the parameters $\left(M_{\star}, R_{\mathrm{e}}, \cdots\right)$ assigned already to the system there may not exist a solution for equation 4.5. In this case another value of $\beta$ is drawn and retried until a solution is found. In some rare cases this effort fails even for some significant number of trials. In such a case we remove the system from the catalog (i.e. reject the whole parameter set assigned). This means that the posterior distribution of $\beta$ can be different from the prior constraint. 

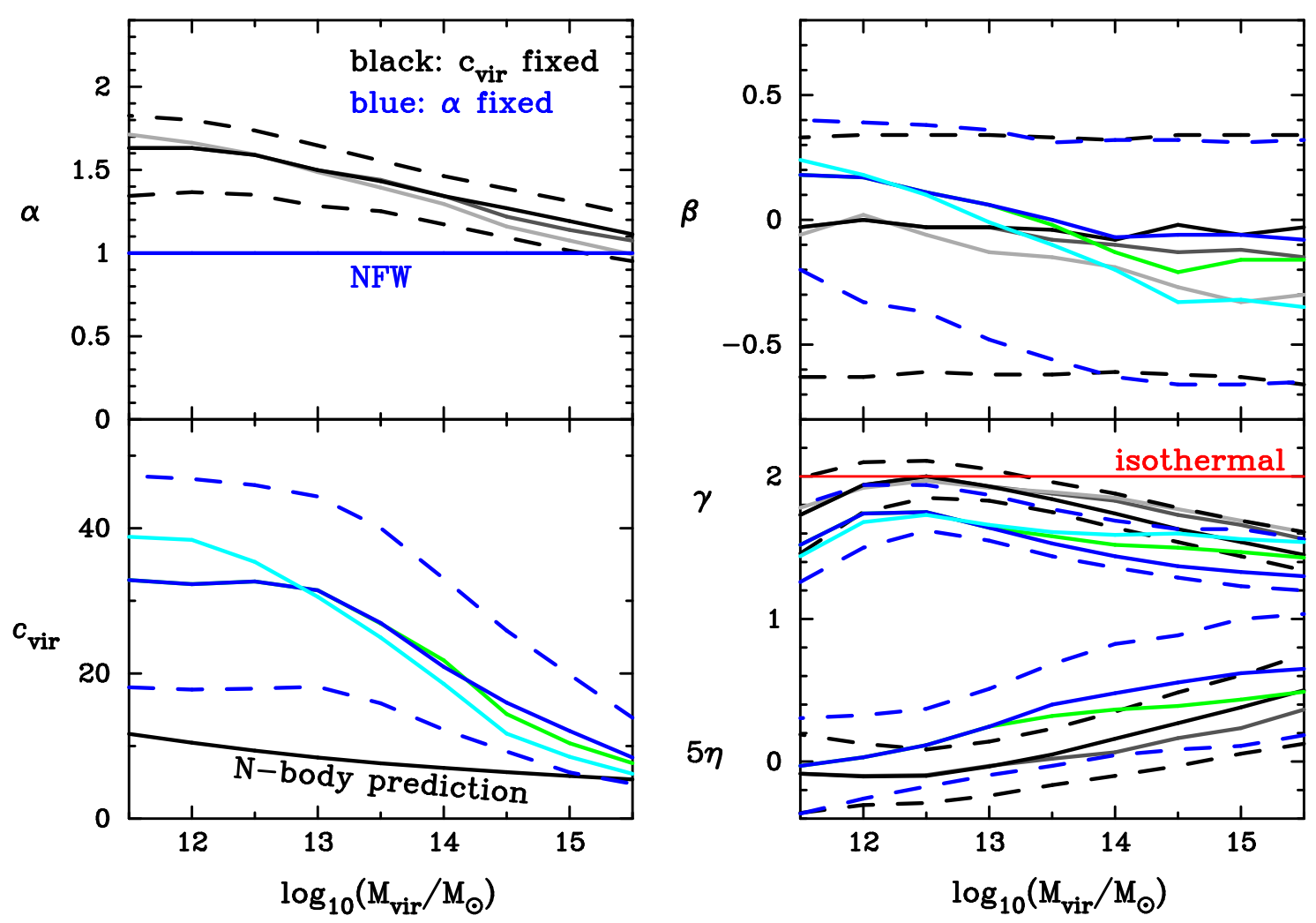

Figure 8. (Left) Distributions of the $\alpha$ NFW halo dark matter density profile parameters $\alpha$ and $c_{\text {vir }}$ with either of them fixed obtained through the spherical Jeans equation based on SDSS velocity dispersions. Solid curves are the median while dashed curves are the $68 \%$ probability limits. The dark gray and green curves are based on corrections of $M_{\star}$ and $\sigma$ due to possible systematic errors of abundance matching for clusters while the light gray and cyan curves include further corrections of stellar masses due to a possible systematic variation of IMF as a function of $\sigma$. (Right) Upper panel shows the simultaneously obtained distribution of constant anisotropy $\beta$ with the prior $[-0.9,0.5]$. Lower panel shows the minus logarithmic slope $\gamma$ of the predicted total (i.e. stellar plus dark matter) mass density profile and the logarithmic slope $\eta$ (multiplied by 5 for visibility) of the velocity dispersion profile at $r=R_{\mathrm{e}} / 2$.

Fig. 8 shows the results for the $\alpha \mathrm{NFW}$ profile as a function of $M_{\mathrm{vir}}$. For the first case $c_{\mathrm{vir}}$ is fixed at the value predicted by the Bolshoi N-body simulation. In this case the resulting values of $\alpha$ lie above the NFW value $\alpha=1$ with increasingly larger values as $M_{\text {vir }}$ decreases. For the second case, $\alpha$ is fixed at the NFW value and $c_{\mathrm{vir}}$ is determined by the Jeans equation. Similarly to the first case the values of $c_{\mathrm{vir}}$ lie above the N-body simulation prediction. These results indicate that dark matter densities are boosted in the inner halos of early-type galaxies. The density boost is greater for a lower mass halo. Qualitatively similar results are obtained for the Einasto profile as well. If $\tilde{\alpha}$ is fixed at 0.17 (the N-body predicted value) [63], the resulting values of $\tilde{c}_{\text {vir }}$ tend to lie above the Bolshoi prediction. If $\tilde{c}_{\text {vir }}$ is fixed at the value predicted by the Bolshoi simulation, the resulting values of $\tilde{\alpha}$ tend to lie below $\tilde{\alpha}=0.17$ implying steepened inner density slopes.

Stellar masses used above are based only on the Chabrier initial mass function (IMF) of 
stars [41]. Recently, there are reports of observational studies that show systematic variations of IMFs across galaxy populations depending on velocity dispersion [68], stellar mass-tolight ratio [69], or color [70]. To quantify systematic effects due to IMFs we consider a systematically varying IMF with velocity dispersion by which stellar masses based on the Chabrier IMF are multiplied by $1.7 \times 10^{1.31 \log _{10}\left(\sigma / \mathrm{km} \mathrm{s}^{-1}\right)-3.14}[68]$ with a constant scatter of 0.15 dex. Furthermore, as shown in Fig. 7 our statistical match between galaxies and halos gives a lower $M_{\star}$ by up to 0.2 dex at fixed $M_{\text {vir }}$ for $M_{\text {vir }}>10^{13} M_{\odot}$ compared with other independent results that are based on IMFs similar to the Chabrier. To gauge possible systematic effects due to stellar mass uncertainties we obtain alternative results taking into account these stellar mass corrections/adjustments. The correction of 0.2 dex in $M_{\star}$ due to abundance matching is accompanied by a corresponding correction in $\sigma$ so as to preserve the observed $M_{\star}-\sigma$ relation. The alternative results are shown in Fig. 8. For the case of using the fixed NFW inner slope $\alpha=1 c_{\mathrm{vir}}$ is lowered by up to $\sim 40$ percent for massive clusters when both systematic effects are applied (cyan curves).

The above two cases (fixing $\alpha / \tilde{\alpha}$ or $c_{\text {vir }} / \tilde{c}_{\text {vir }}$ ) imply different inner density profiles that cannot be distinguished by the SDSS velocity dispersion alone. It shows the degeneracy of the allowed density profiles given only the parameters of a system in the SEC described above (section 3.2). In fact, the degeneracy is broader than the above two cases. In other words, other combinations of $\alpha$ and $c_{\text {vir }}$ (or, $\tilde{\alpha}$ and $\tilde{c}_{\text {vir }}$ ) can satisfy the Jeans equation as well. To break the degeneracy we need additional constraints. Those constraints may also help to constrain the velocity dispersion anisotropy.

The upper right panel of Fig. 8 shows the posterior distributions of constant velocity dispersion anisotropy $\beta$. In some cases (mostly the cases of fixing $\alpha=1$ ) the posterior distributions are clearly different from the prior distribution given by equation (4.14). As mentioned above, this occurs because only certain combinations of $\left\{\alpha / \tilde{\alpha}, c_{\mathrm{vir}} / \tilde{c}_{\mathrm{vir}}, \beta\right\}$ can satisfy the Jeans equation for a system whose other parameters have been specified in advance and a randomly selected $\beta$ may or may not be a solution.

The bottom right panel of Fig. 8 shows the distributions of the negative logarithmic slope $\gamma$ of the three-dimensional total radial density profile at $R_{\mathrm{e}} / 2$, i.e. $\gamma \equiv-d \ln \left[\rho_{\star}(r)+\right.$ $\left.\rho_{\mathrm{dm}}(r)\right] / d \ln r$. The two cases give the values of $\gamma$ offset by $\approx 0.2-0.3$. Fig. 8 also shows the distributions of the logarithmic slope $\eta$ (see section 4.3.2) of the velocity dispersion profile at $R_{\mathrm{e}} / 2$. There is also an offset of $\approx 0.02-0.07$ in $\eta$ between the two cases. The distributions of $\gamma$ and $\eta$ in the two cases shown in Fig. 8 hint that dark matter density boost in the inner halo is more consistent with enhanced $\alpha$ at fixed $c_{\text {vir }}$ than enhanced $c_{\text {vir }}$ at fixed $\alpha$ because the former case gives distributions of $\gamma$ and $\eta$ that are more consistent with current gravitational lensing [9-11] and spectroscopic $[8,71]$ observations of early-type galaxies. We consider below (section 4.3.2) incorporating spectroscopic observations into our SEC through Jeans modeling.

\subsubsection{Dark matter density profiles statistically matched with velocity dispersion profiles: completion of the semi-empirical catalog}

The SDSS velocity dispersion corresponds to the luminosity-weighted LOSVD within $R_{\mathrm{e}} / 8$. Clearly, this alone cannot constrain uniquely the dark matter distribution in the halo. Measured velocity dispersions at multiple radii for each SDSS galaxy would be most useful in breaking the degeneracy. Without them we consider independent observational constraints on the velocity dispersion profile (VP) of early-type galaxies $[8,71]$. The available observational constraints show that the logarithmic slope $\eta$ of the luminosity-weighted LOSVD 
profile at $R_{\mathrm{e}} / 2$, i.e. $\eta \equiv d \ln \left\langle\sigma_{\text {los }}\right\rangle(R) / d \ln R$, ranges from $-0.066 \pm 0.035$ [8] to -0.04 [71]. We assume that the mean value of $\eta$ is given by the mean of these two independent measurements, i.e., $\langle\eta\rangle=-0.053$. For the dispersion of $\eta$ we assume the root-mean-square of the measured dispersion 0.035 for one sample and the difference between the two independent means 0.026 , i.e., $\sigma_{\eta}=0.044$.

We then proceed as follows. For each system in the SEC we first obtain a degenerate set of $\left\{\alpha, c_{\mathrm{vir}}, \beta\right\}$. We do this by assigning random values to $\alpha$ and $\beta$ and then solving for $c_{\mathrm{vir}}$ using equation (4.5). We assume the priors $0.1<\alpha<2.5$ and equation (4.14) for $\alpha$ and $\beta$ respectively. We then select one out of this degenerate set for each system using a selection function $S(\eta)$ so that the posterior distribution of $\eta$ for all systems in the SEC matches the observed distribution described above. An uniform selection function would result in a posterior distribution of $\eta$ that is biased toward a shallower VP (i.e. $\eta>-0.53$ ). After numerical experiments we choose the following selection function

$$
S(x) \propto\left\{\begin{array}{cl}
{[1-\theta(x-\mu)] \exp \left[-\frac{(x-\mu)^{2}}{2 \sigma_{\mathrm{L}}^{2}}\right]+\theta(x-\mu) \exp \left[-\frac{(x-\mu)^{2}}{2 \sigma_{\mathrm{H}}^{2}}\right]} & \text { if } x>-0.2, \\
0 & \text { else }
\end{array}\right.
$$

where $\mu=-0.066, \sigma_{\mathrm{L}}=0.1, \sigma_{\mathrm{H}}=0.01$ and $\theta(x)$ is the theta function. Notice that the above selection function has the peculiar property that the most probable value is displaced downward and the upward width is negligibly small. Any selection function having these features would do the same job. After selecting an $x$ using this selection function we select one set $\left\{\alpha, c_{\mathrm{vir}}, \beta\right\}$ (out of the above degenerate set for the given system in the SEC) that best matches $x$ by minimizing the figure-of-merit function defined by

$$
Q_{(\mathrm{VP})}^{2} \equiv\left(\frac{\eta-x}{\sigma_{\eta}}\right)^{2}
$$

If the value of $Q_{(\mathrm{VP})}^{2}$ is greater than 13.7 for the best-fit set, then the whole degenerate set is rejected. This criterion matters only for $M_{\text {vir }} \gtrsim 10^{13.5} \mathrm{M}_{\odot}$ and is chosen so that the posterior distribution of $\eta$ deviates minimally from our adopted observational constraint of $\eta=-0.053 \pm 0.044$ while not causing any bias in the distributions of other parameters in the SEC. A criterion lower (i.e. stronger) than 13.7 would bring the posterior distribution of $\eta$ closer to the observational constraint but the resulting systems tend to have biased distributions of $R_{\mathrm{e}}$ and $n$. A weaker criterion would allow $\eta$ to deviate more. Our assumption is the minimal deviation. Because of this difficulty our procedure becomes less reliable as $M_{\text {vir }}$ increases toward massive clusters.

Fig. 9 shows the posterior distribution of $\eta$. The mean value agrees well with the input empirical mean for $M_{\text {vir }} \lesssim 10^{13.5} \mathrm{M}_{\odot}$ but deviates upward systematically as $M_{\text {vir }}$ increases. As mentioned above this deviation is unavoidable without biasing the distributions of observed galaxy parameters such as $R_{\mathrm{e}}$. In this sens our results require the VP slope to vary systematically with $M_{\mathrm{vir}}$. This deviation does not necessarily mean a discrepancy between our results and the observed VPs because the latter $[8,71]$ cannot be used to address any systematic variation that may be present. The galaxy-to-galaxy dispersion of $\eta$ is somewhat lower than the adopted $\sigma_{\eta}=0.044$ for $M_{\text {vir }} \lesssim 10^{13.5} \mathrm{M}_{\odot}$ but higher for $M_{\text {vir }} \gtrsim 10^{13.5} \mathrm{M}_{\odot}$. Note, however, that $\sigma_{\eta}=0.044$ includes a systematic error between the two samples used (see above). Our dispersion is more consistent with the intrinsic scatter of 0.035 in one sample [8]. 


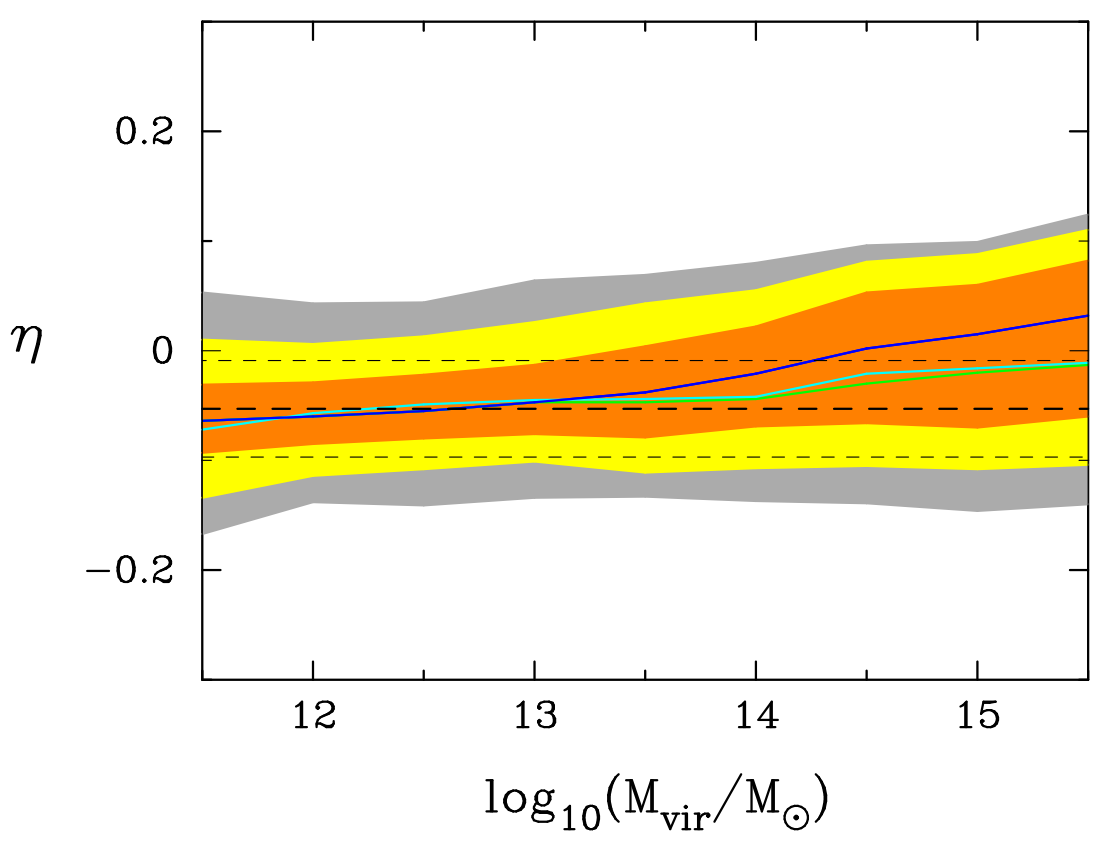

Figure 9. The posterior distribution of $\eta$ (the VP slope at $R_{\mathrm{e}} / 2$ ) for our early-type systems is compared with the input observational constraint of $\eta=-0.053 \pm 0.044$ (black dashed lines). The blue solid curve is the expectation value while the orange, yellow and gray regions contain respectively $68 \%, 95 \%$, and $99.7 \%$ of the systems. The green and cyan curves are for the corrected stellar masses as in Fig. 8. These results are based on Jeans dynamical modeling of our systems in the SEC using the observational constraint on $\eta$ as a statistical input.

Through the above procedure we have effectively assigned dark matter density profile, velocity dispersion profile slope at $R_{\mathrm{e}} / 2$ and constant velocity dispersion anisotropy simultaneously to each system in the SEC so that the Jeans equation is satisfied along with all the adopted observational constraints. We have thus determined all the unknowns of the SEC making it a complete catalog of early-type galaxy-halo systems.

Fig. 10 shows the distribution of the dark matter density profiles based on the $\alpha \mathrm{NFW}$ model in the final SEC. The distributions of $\alpha$ and $c_{\mathrm{vir}}$ show that their expectation values are varying systematically with $M_{\text {vir }}$ and there are significant halo-to-halo variations at fixed $M_{\mathrm{vir}}$. The expectation values of $\alpha$ lie above the NFW value for $M_{\mathrm{vir}} \lesssim 10^{13.5-14.5} \mathrm{M}_{\odot}$ and are typically $\langle\alpha\rangle \approx 1.3$ for galactic halos with $10^{12} \mathrm{M}_{\odot} \lesssim M_{\text {vir }} \lesssim 10^{13-14} \mathrm{M}_{\odot}$. The halo-to-halo root mean square $(\mathrm{rms})$ scatter is typically $\operatorname{rms}(\alpha) \sim 0.4-0.5$. Hence for our $\sim 1000$ halos at fixed $M_{\text {vir }}$ the estimated error of $\langle\alpha\rangle$ is $\sim 0.015$ implying that $\langle\alpha\rangle \approx 1.3$ for $10^{12} \mathrm{M}_{\odot} \lesssim M_{\text {vir }} \lesssim 10^{13-14} \mathrm{M}_{\odot}$ is some $20 \sigma$ above the NFW value $\alpha=1$. The NFW profile is clearly ruled out as a mean profile of galactic-scale halos implying significant effects of halo contraction. The trend of $c_{\text {vir }}$ follows overall the N-body prediction $[44,53]$. The expectation values are within $20 \%$ (systematic error) of the N-body prediction except for low mass galactic halos with $M_{\text {vir }} \lesssim 10^{12} \mathrm{M}_{\odot}$ for which the expectation values are clearly higher.

Fig. 10 also shows the distribution of the negative logarithmic slope $\gamma$ at $R_{\mathrm{e}} / 2$ for the total mass distribution. For halos with $10^{12} \mathrm{M}_{\odot} \lesssim M_{\mathrm{vir}} \lesssim 10^{14} \mathrm{M}_{\odot}$ the values of $\gamma$ scatter around the isothermal value $\gamma=2$ with the expectation value of $1.9 \lesssim\langle\gamma\rangle \lesssim 2.1$ and the rms 

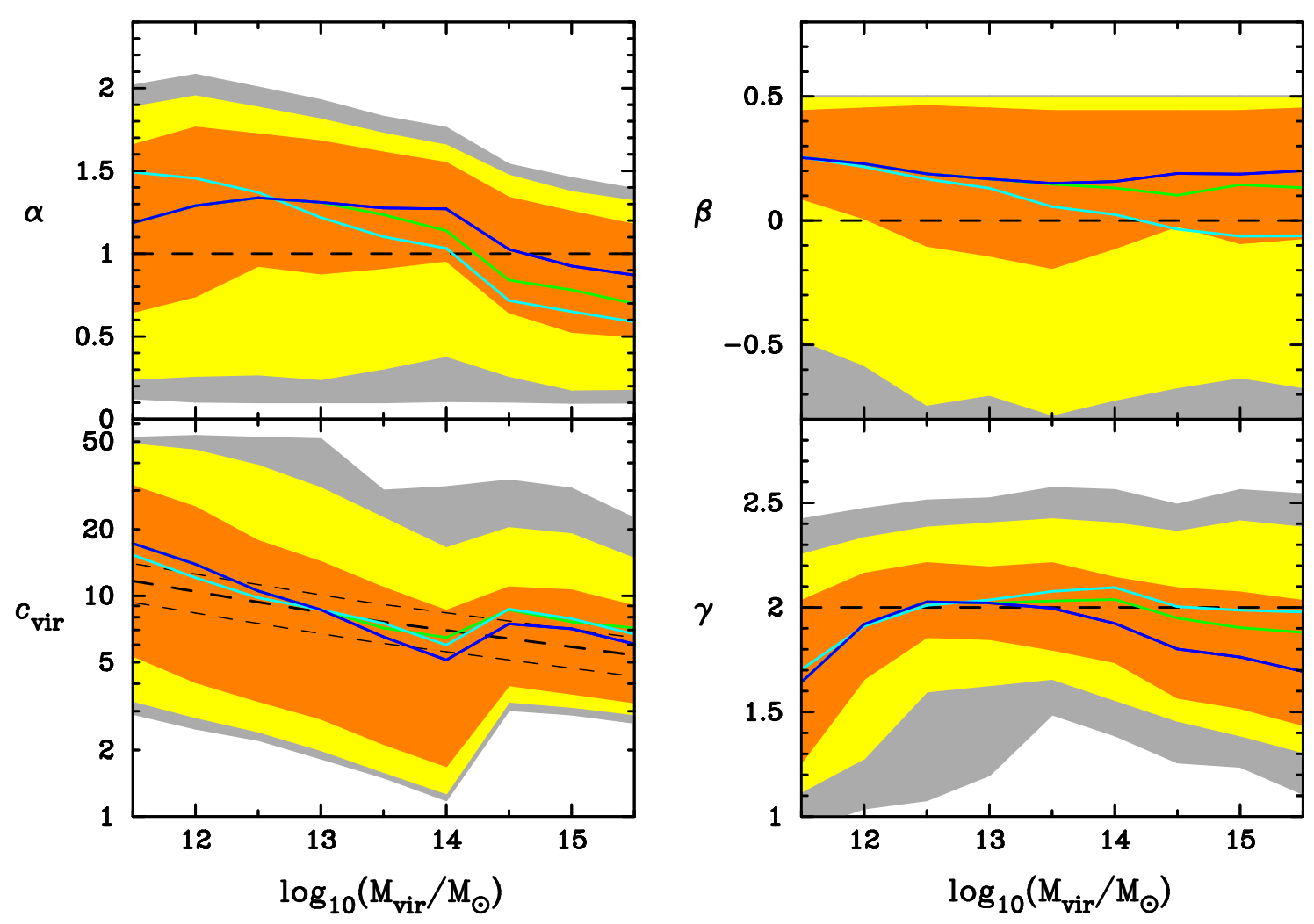

Figure 10. Distributions of the same parameters of Fig. 8, i.e. $\alpha$ and $c_{\text {vir }}$, in our final SEC for which each system is assigned a velocity dispersion profile satisfying the spherical Jeans equation. The blue solid curves show the expectation values. The green and cyan solid curves are for the corrected stellar masses as in Fig. 8. Orange, yellow and gray regions contain the 68\%, 95\% and $99.7 \%$ occurrences respectively. The black dashed line in the lower left panel shows the mean NFW concentration predicted by the Bolshoi N-body simulation with thin dashed lines showing a systematic error of $20 \%$. The black dashed line in the lower right panel corresponds to the isothermal profile.

galaxy-to-galaxy scatter of $\operatorname{rms}(\alpha) \approx 0.2-0.3$. This result for a general sample of early-type galaxies is in good agreement with the results for strong lensing observations of early-type galaxies [9-11]. For massive cluster-sized halos the central total density profile within the optical region tends to be shallower than the isothermal for stellar masses based on the Chabrier IMF but becomes close to the isothermal for stellar masses based on a systematic variation of IMF as a function of $\sigma$. The distribution of velocity dispersion anisotropy $\beta$ is tilted toward a mild radial anisotropy with the expectation value close to isotropy. This distribution is consistent with independent results for dozens of individual systems $[6,66,67]$.

Fig. 11 shows the corresponding results for the Einasto model. The distributions of $\beta$ and $\gamma$ are strikingly similar to those for the $\alpha$ NFW model. The distributions of $\tilde{\alpha}$ and $\tilde{c}_{\text {vir }}$ cannot be directly compared with those of $\alpha$ and $c_{\mathrm{vir}}$. For the Einasto model the logarithmic slope of the density varies continuously toward the origin, i.e. $\gamma_{\mathrm{dm}}=2\left(\tilde{c}_{\mathrm{vir}} r / r_{\mathrm{vir}}\right)^{\tilde{\alpha}}$ for $\rho_{\mathrm{dm}}(r) \propto r^{-\gamma_{\mathrm{dm}}}$, so that unlike the $\alpha \mathrm{NFW}$ model an inner density slope cannot be characterized by a single parameter. At a fixed inner radius the slope $\gamma_{\mathrm{dm}}$ can be boosted either by increasing $\tilde{c}_{\mathrm{vir}}$ 

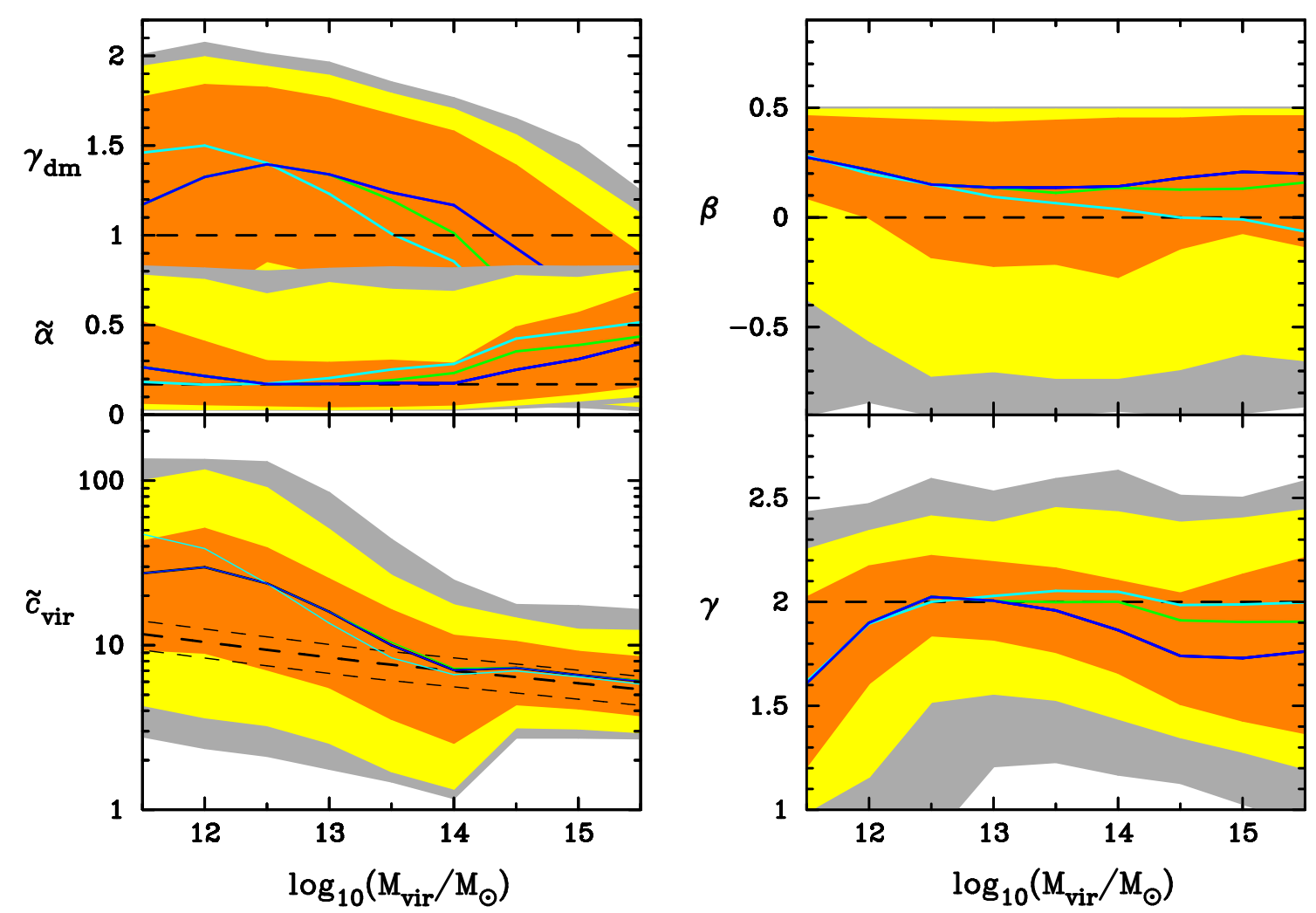

Figure 11. Same as Fig. 10 except that the Einasto model parameters $\tilde{\alpha}$ and $\tilde{c}_{\text {vir }}$ are shown. Parameter $\gamma_{\mathrm{dm}}$ is the minus logarithmic slope for the Einasto model dark matter distribution, i.e. $\gamma_{\mathrm{dm}} \equiv-d \ln \rho_{\operatorname{Ein}}(r) / d \ln r$ at $R_{\mathrm{e}} / 2$. The constrained values of $\gamma_{\mathrm{dm}}$ match well with those of $\alpha$ of the $\alpha \mathrm{NFW}$ model shown in Fig. 10.

or decreasing $\tilde{\alpha}$. Fig. 11 indicates that the former is more likely the case for galactic halos. Namely, for $M_{\text {vir }} \lesssim 10^{13-14} \mathrm{M}_{\odot}\langle\tilde{\alpha}\rangle \sim 0.17$ with $\left\langle\tilde{c}_{\mathrm{vir}}\right\rangle$ greater than the N-body prediction. However, for cluster halos $\left\langle\tilde{c}_{\mathrm{vir}}\right\rangle$ is within $20 \%$ of the N-body prediction while $\langle\alpha\rangle$ tends to deviate from 0.17. The distribution of $\gamma_{\mathrm{dm}}$ at $R_{\mathrm{e}} / 2$ is similar to that of $\alpha$ of the $\alpha \mathrm{NFW}$ model.

We have tested our systems against a couple of dynamical mass scaling relations presented recently in the literature. One scaling involves the mass within $r \sim R_{\mathrm{e}} / 2$ and the LOSVD at that radius [72] while the other scaling involves the mass within $r \approx 4 R_{\mathrm{e}} / 3$ and the luminosity-weighted LOSVD to infinity [73]. For our systems the average masses predicted by these scalings are accurate within $10 \%$ for $10^{12} \mathrm{M}_{\odot} \lesssim M_{\text {vir }} \lesssim 10^{14} \mathrm{M}_{\odot}$ and there are system-to-system variations with a typical dispersion of $5 \%$ to $10 \%$ at fixed $M_{\text {vir }}$ (appendix D). 


\section{Implication for baryon-induced halo contraction and characterization of the contracted profiles}

The distribution of dark matter density profiles in our final semi-empirical catalog (SEC) of early-type systems supports the hypothesis that halos are modified in response to dissipational gas cooling and galaxy formation $[39,40]$. Our results indicate that galactic halos with $M_{\text {vir }} \lesssim 10^{13.5} \mathrm{M}_{\odot}$, on average, have contracted and dark matter density profiles of the contracted halos are diverse. Fig. 8 and Fig. 10 show that halo contraction is more likely to be realized by steepening of inner density slope $(\alpha)$ rather than decrease of scale radius (i.e. increase of concentration $c_{\mathrm{vir}}$ ) as the former is more consistent with the current observational constraints on the velocity dispersion profile of early-type galaxies.

To quantify halo contraction we consider the ratios of the dark matter masses of our halos to those of NFW halos with the average concentrations predicted by the Bolshoi dissipationless N-body simulation within three radii, i.e. the effective radius $R_{\mathrm{e}}$, the NFW scale radius $r_{\mathrm{s}, \mathrm{NFW}}$ and one fifth of the halo virial radius $\left(r_{\mathrm{vir}} / 5\right)$. If the NFW profile were assumed for the initial N-body predicted halo and there were no modification to the halo due to baryonic effects, then these ratios should center around unity with scatters arising solely from the scatters of N-body predicted NFW concentrations.

The calculated ratios are displayed in Fig. 12. It shows evidently dark matter density enhancement in the optical region of the inner halo. The mean density enhancement factor within $r=R_{\mathrm{e}}$ increases steeply from $\approx 1$ (no enhancement) at $M_{\mathrm{vir}} \approx 10^{15-15.5} \mathrm{M}_{\odot}$ (or $\left.10^{13.5-14} \mathrm{M}_{\odot}\right)$ up to $3-4$ at $M_{\text {vir }} \approx 10^{12} \mathrm{M}_{\odot}$. As $M_{\text {vir }}$ decreases, however, the scatter also increases rapidly indicating greater diversity. The Kolmogorov-Smirnov (K-S) tests of the distributions or the Student t tests of the means at fixed $M_{\text {vir }}$ rule out the null hypothesis that halo profiles follow the dissipationless N-body predictions with a typical dispersion of 0.14 dex in $c_{\mathrm{vir}}$ [74] even after allowing for a systematic error of $20 \%$ in the mean concentrations (K-S and t probabilities $\lesssim 10^{-5}$ ). The K-S and t tests show that our halo profiles can be consistent with the N-body predictions at $M_{\mathrm{vir}} \approx 10^{15-15.5} \mathrm{M}_{\odot}$ if the Chabrier IMF is assumed but at $10^{13.5-14} \mathrm{M}_{\odot}$ if the IMF varies systematically with stellar velocity dispersion [68]. Interestingly, the dark matter mass within $r=R_{\mathrm{e}}$ for our halos based on the systematically varying IMF is lower than the N-body prediction for $M_{\text {vir }} \gtrsim$ $10^{14.5} \mathrm{M}_{\odot}$ suggesting a possibility of inner halo expansion in those massive clusters. However, this possibility of halo expansion for massive clusters should be taken with caution as our procedure is less reliable for massive clusters (see section 4.3.2) due to uncertainties in velocity dispersion profiles and stellar masses.

At the scale of $r_{\mathrm{s}, \mathrm{NFW}}$ (i.e. the N-body predicted scale radius assuming the NFW model) the halo density enhancement is weakly present only for $M_{\text {vir }} \lesssim 10^{13} \mathrm{M}_{\odot}$. At the scale of $r_{\text {vir }} / 5$ the mean density enhancement is $\approx 20 \%$ at best for galactic halos. A statistical analysis of weak lensing effects for $r \geq r_{\text {vir }} / 5$ based on statistically representative samples of galaxies, groups and clusters from SDSS yields concentrations similar to N-body predictions assuming the NFW profile for $10^{12} \mathrm{M}_{\odot} \lesssim M_{\text {vir }} \lesssim 10^{15} \mathrm{M}_{\odot}$ [75]. This result is consistent with our finding of no or little density enhancement within $r=r_{\mathrm{vir}} / 5$. The weak lensing measured scatter of concentration gives rise to $1 \sigma$ scatter of $10 \%-20 \%$ in mass within $r=r_{\mathrm{vir}} / 5$ consistent with our result shown in Fig. 12.

Fig. 12 also shows the fraction of dark matter mass within a sphere of radius $r=R_{\mathrm{e}}$ for our halos embedding early-type galaxies. The mean dark matter fraction increases with $M_{\text {vir }}$ for the case of Chabier IMF but decreases for the case of the systematically varying 

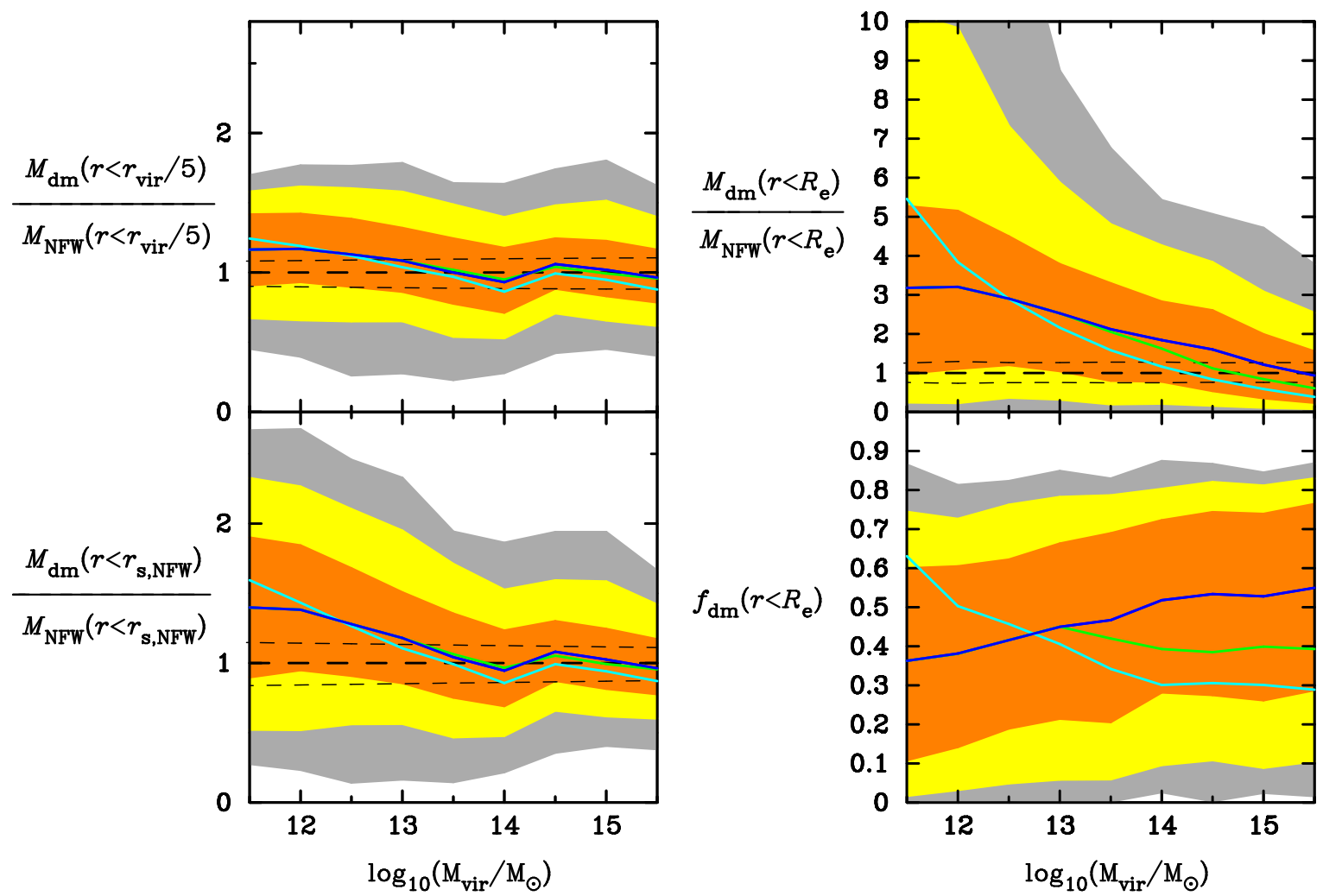

Figure 12. Dark matter masses and fractions based on our Jeans dynamical modeling results for the $\alpha$ NFW model. Color representations of lines and regions are the same as in Fig. 10. The corresponding results for the Einasto model are similar to those shown here. (Left panels \& Top Right) Ratio of the dark matter masses within a certain three dimensional radius for the initial galaxy-less NFW halo $\left(M_{\mathrm{NFW}}\right)$ and the realistic halo embedding an early-type galaxy at the center $\left(M_{\mathrm{dm}}\right)$. Here $r_{\mathrm{s}, \mathrm{NFW}}$ is the scale radius of the NFW profile while $R_{\mathrm{e}}$ is the effective radius of the embedded central galaxy. The black thick dashed line is the expected mean (i.e. unity) for the case of no modification of the halo while thin dashed lines show $20 \%$ systematic error in the N-body predicted $c_{\mathrm{vir}}$. These results show that most halos have contracted in the optical region of central galaxies with the varying degree of mass enhancement depending on the the halo virial mass. However, the contraction has modified the halo up to the scale radius only for relatively less massive halos with $M_{\text {vir }} \lesssim 10^{13} \mathrm{M}_{\odot}$ and starts to be unimportant beyond the scale of $20 \%$ of the virial radius. (Bottom Right) Fraction of dark matter mass within $R_{\mathrm{e}}$ of the early-type galaxy embedded at the center of a halo.

IMF. The mean fraction lies 0.2 to 0.5 for $10^{12} \mathrm{M}_{\odot} \lesssim M_{\text {vir }} \lesssim 10^{14} \mathrm{M}_{\odot}$ depending on $M_{\text {vir }}$ and the assumption of IMF. Dark matter fraction at fixed $M_{\text {vir }}$ covers a broad range showing a great diversity. Dynamical analyses of nearby elliptical galaxies indicate $\sim 30 \%$ of dark matter contribution within $R_{\mathrm{e}}[66]$ and a recent analysis of strong lensing galaxies indicates that $f_{\mathrm{dm}}\left(r<R_{\mathrm{e}}\right)$ can be $\sim 0.2-0.6$ [11]. These results agree well with our results.

Traditionally, adiabatic contraction has been invoked for the response of halos to dissipational baryonic processes $[24,25,39,40]$. Hydrodynamic simulations have been used to study adiabatic contraction in detail recently [24, 25]. Independent of hydrodynamic simulations and relying only on SDSS observed galaxy data and robust classical results from 
N-body dissipationless simulations, our results demonstrate evidently that halo contraction takes place in most occurrences of spheroidal galaxy formation.

Contraction of halo mass profile in response to the baryon condensation by itself is expected to lead to steepening of the central density profile out to $\sim 5-10 \%$ of the virial radius but leave the outer mass profile intact [40]. However, hierarchical formation of central galaxy may also result in an additional mild increase of halo concentration due to several other processes [76]. Our results are in line with these expectations.

An observational indication of adiabatic contraction in a statistically representative sample of SDSS elliptical galaxies is reported recently [20] based on a combination of weak lensing measurements of halos and stellar velocity dispersions. These authors assume the NFW profile and notice that their analysis requires higher concentrations of halos than pure dark matter simulation halos. A combined analysis of strong and weak lensing effects for 28 clusters from the Sloan Giant Arcs Survey finds increasingly over-concentration as halo mass gets lower assuming the NFW profile [23].

Based on generalized dark matter density profiles that are allowed to vary from the NFW profile, our results provide not only (semi-empirical) evidence for halo contraction but also a characterization of contraction. Our results indicate that contracted halo profiles generally deviate from the NFW and display a range of diversity and halo contraction is more frequently realized by steepening of the radial density slope rather than rescaling of the NFW profile. If the NFW profile were forcibly used to describe the contracted halo, then the concentration would be significantly higher than that of the pure dark matter simulation halo which would match high concentrations of halos obtained by recent observational studies assuming the NFW profile $[20,23]$.

Our analysis presented here relies on N-body simulation data as well as observed galaxy data. In this sense our results are referred to as semi-empirical. It would be of great importance to carry out a similar but fully empirical analysis that relies only on empirical information. A development of such an analysis is under way and will be presented in the near future. However, our current analysis has the advantage that we can naturally construct a mock universe into a N-body simulation lightcone. Such a mock universe will be particularly useful for realistic strong lensing simulation for future surveys because baryonic effect is a crucial factor for strong lensing.

It would be useful to carry out a semi-empirical (as was done here) or fully empirical analysis for other morphological types of galaxies. An analysis based on empirical information (satellite kinematics and weak lensing observations of halos and observed galaxy scaling relations) and N-body simulation results indicates that halos may expand in response to baryonic effects for late-type systems [30]. Recently, $\Lambda$ CDM N-body + SPH (smoothed particle hydrodynamic) simulations of low-mass systems with $M_{\mathrm{vir}} \lesssim 10^{11.5} \mathrm{M}_{\odot}\left(M_{\star} \lesssim 10^{9.4} \mathrm{M}_{\odot}\right)$ show that supernovae driven outflows can transfer enough energy to dark matter particles so that dark halo expands transforming initially cuspy central profile into a shallower core [77]. Another recent cosmological hydrodynamic simulation of a massive spiral galaxy with $M_{\mathrm{vir}} \approx 10^{12} \mathrm{M}_{\odot}$ also argues for halo expansion [78].

The literature results of halo expansion for late-type and dwarf systems contrast strikingly with our results of halo contraction for early-type systems. This apparent contrast is already noticed and discussed in the literature [30]. If this contrast is real (i.e. both expansion and contraction results are correct), merging driven galaxy transformation [79] may play the vital role in creating contracted halos of early-type galaxies. Also, our finding that the degree of halo contraction for early-type galaxies is a decreasing function of halo mass 
indicates that the effects of merging depend on various factors such as morphological types of merging subunits, the amount of gas (i.e. whether wet or dry) and perhaps AGN driven outflows from the central supermassive black holes. However, before interpreting halo expansion/contraction it is more important to address thoroughly possible systematic errors of our and literature results. Possible systematic errors of our results include the distribution of stellar IMFs, velocity dispersion anisotropy shapes, velocity dispersion profiles, the N-body simulation produced halo mass function, and the spherical mass models (the real early-type systems are ellipsoidal in general). We have addressed the first three of these possible errors here although they can be (and should be) better addressed with better empirical information in the future. The use of abundance matching has also been checked against empirical halo mass-stellar mass relations and its possible systematic error for large halo mass has been addressed. However, we have not yet addressed the effect of non-sphericity of mass models. An optimistic prediction is that oblate-like and prolate-like shapes are equally likely so that their effects cancel statistically. To quantify the effect of non-sphericity non-spherical Jeans equations need to be used in the future based on empirical information on shapes. With regard to hydrodynamic simulation results for halo expansion in the literature empirical methods should also be used in the future to test those results independently and (more importantly) empirically.

\section{Characterizing dark matter annihilation strength in the halos of early- type galaxies}

One proposed way of identifying dark matter particles has been the detection of $\gamma$-ray emission from dark matter annihilation or decay in the halos $[19,80]$. In the case of dark matter annihilation the predicted $\gamma$-ray flux is known to be very sensitive to dark matter density. Specifically, the predicted $\gamma$-ray flux from dark matter annihilation is proportional to the dark matter density squared integrated along the line-of-sight. When averaged over the angular scale of the halo it can be approximated by [81]

$$
J_{\mathrm{dm}} \simeq \frac{1}{D^{2}} \int_{\mathrm{Vol}} r^{2} \rho_{\mathrm{dm}}^{2}(r) d r
$$

where $D$ is the distance to the halo.

Clearly, halo contraction is expected to boost the predicted $\gamma$-ray flux from dark matter annihilation. Fig. 13 shows the predicted values of $J_{\mathrm{dm}} \times D^{2}$ for our constrained halo dark matter distributions based on the Einasto model. The $\alpha \mathrm{NFW}$ model cannot be used for calculating $J_{\mathrm{dm}}$ because $J_{\mathrm{dm}}$ diverges for $\alpha>1.5$ as $r \rightarrow 0$. The specific values of $J_{\mathrm{dm}}$ at a fiducial distance of $D=d_{4} 4 \mathrm{Mpc}$ are also shown and compared against several local dwarf spheroidal galaxies [82] and low redshift clusters [81] that have been proposed as promising targets for $\gamma$-ray flux. Fig. 13 suggests that typical nearby early-type galaxies with $M_{\text {vir }} \sim 10^{12} \mathrm{M}_{\odot}-10^{13} \mathrm{M}_{\odot}$ can also be promising targets. This rather surprising prediction is the consequence of the mass dependent enhancement of $J_{\mathrm{dm}}$ as shown in the middle panel of Fig. 13, resulting from the varying degree of halo contraction. We note that there are two well-known elliptical or lenticular galaxies at a distance of $\sim 4 \mathrm{Mpc}$, Centaurus A (NGC 5128) and Maffei 1. Although our results are only for the halos embedding early-type galaxies at their centers, they highlight the importance of halo contraction induced by galaxy formation for dark matter search. 


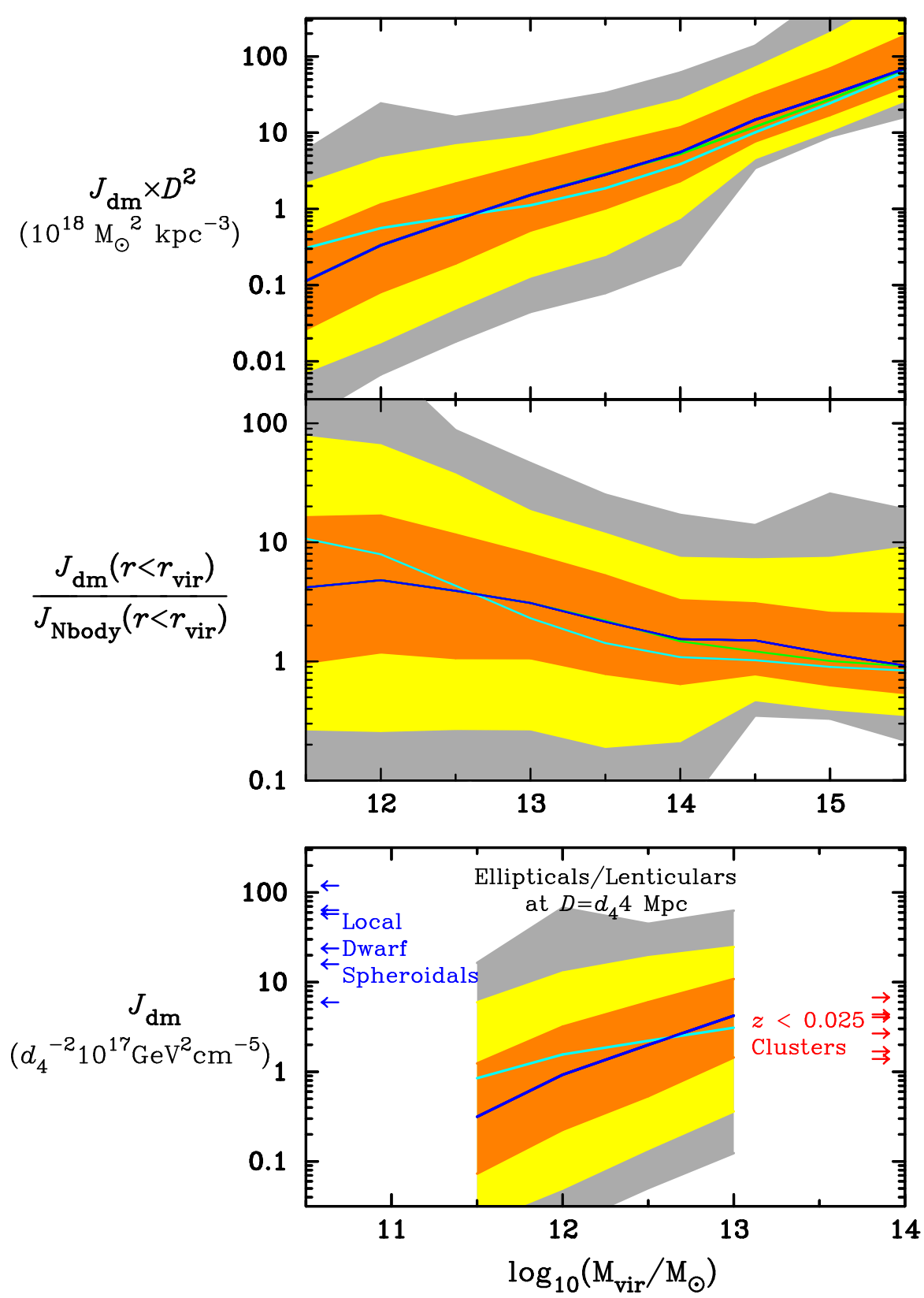

Figure 13. (Top) The dark matter density squared integrated along the line-of-sight and averaged on the halo angular scale, $J_{\mathrm{dm}}$ times the halo distance squared $D^{2}$, approximated by $J_{\mathrm{dm}} D^{2} \simeq \int r^{2} \rho^{2}(r) d r$ [81] for the halos in our final SEC. The results are shown only for the Einasto model because $J_{\mathrm{dm}}$ diverges for certain central cusps of the $\alpha \mathrm{NFW}$ model. The predicted $\gamma$-ray flux from dark matter annihilation is proportional to $J_{\mathrm{dm}}$. (Middle) Ratio of $J_{\mathrm{dm}}$ for the halo embedding a central early-type galaxy to that for the dark matter only N-body simulation halo with $\tilde{\alpha}=0.17$ and $\tilde{c}_{\text {vir }}$ matched to the Bolshoi concentrations. This shows that the predicted $\gamma$-ray flux is enhanced in most galactic halos embedding spheroidal galaxies. (Bottom) The predicted range of $J_{\mathrm{dm}}$ for nearby normal spheroidals (i.e. ellipticals and lenticulars) at a fiducial distance of $D=d_{4} 4 \mathrm{Mpc}$ is shown and compared with the estimates for local dwarf spheroidal galaxies (blue arrows) [82] and low-redshift $(z<0.025)$ clusters (red arrows) [81]. 


\section{Conclusions}

We have constructed a semi-empirical catalog of early-type galaxy-halo systems through a combination of the observed statistical properties of SDSS galaxies and the halo mass function from the Bolshoi N-body dissipationless simulation in conjunction with the observed properties of velocity dispersion profiles of early-type galaxies. In constructing the catalog we have determined dark matter density profiles through Jeans dynamical modeling. Based on the systems in our catalog we find the following statistical properties for early-type galaxies and their embedding halos:

- The distribution of total mass density profiles within the effective radius at fixed $M_{\mathrm{vir}}$ scatters around the isothermal profile for $10^{12} \mathrm{M}_{\odot} \lesssim M_{\text {vir }} \lesssim 10^{14} \mathrm{M}_{\odot}$. The density slope $\gamma$ at $r=R_{\mathrm{e}} / 2$ for $\rho(r) \propto r^{-\gamma}$ has a mean value $1.9 \lesssim\langle\gamma\rangle \lesssim 2.1$ with a rms scatter of $\approx 0.2-0.3$.

- The inferred dark matter density profiles of the halos imply significantly higher dark matter densities in the inner regions compared with those from the dissipationless simulation. The mean density boost factor within a sphere of $R_{\mathrm{e}}$ ranges from $\approx 1$ for $M_{\mathrm{vir}} \approx 10^{15-15.5} \mathrm{M}_{\odot}\left(\right.$ or $\left.10^{13.5-14} \mathrm{M}_{\odot}\right)$ to $\approx 3-4$ for $M_{\mathrm{vir}} \approx 10^{12} \mathrm{M}_{\odot}$. This provides an independent support for halo contraction for galactic halos.

- The inferred mean dark matter densities within a sphere of $r_{\mathrm{vir}} / 5$ are at most $\approx 20 \%$ higher than the N-body prediction implying that the outer mass profiles are minimally affected by halo contraction.

- Using a couple of general-class models for the dark matter distribution we obtain statistical characterizations of the contracted profiles. For galactic halos with $10^{12} \mathrm{M}_{\odot} \lesssim$ $M_{\text {vir }} \lesssim 10^{13} \mathrm{M}_{\odot}$, the three-dimensional dark matter density slope $\gamma_{\mathrm{dm}}$ at $r=R_{\mathrm{e}} / 2$ for $\rho_{\mathrm{dm}}(r) \propto r^{-\gamma_{\mathrm{dm}}}$ has a mean value of $1.2 \lesssim\left\langle\gamma_{\mathrm{dm}}\right\rangle \lesssim 1.4$ with a rms scatter of $\approx 0.4-0.5$. The NFW profile is clearly ruled out as a mean profile although some fraction of halos may well follow it.

- The dark matter fraction within the sphere of radius $R_{\mathrm{e}}$ has a mean value ranging from 0.2 to 0.5 with a typical rms scatter of $\approx 0.2-0.3$ for $10^{12} \mathrm{M}_{\odot} \lesssim M_{\text {vir }} \lesssim 10^{15} \mathrm{M}_{\odot}$.

- Halo contraction boosts significantly dark matter annihilation strength in the halos embedding early-type galaxies so that nearby early-type galaxies may be promising targets for indirect dark matter search.

\section{Acknowledgments}

We would like to thank Dan Hooper for conversations and comments on the draft and Anatoly Klypin for making outputs from the Bolshoi and Multidark simulations available to us. We would also like to thank the anonymous referees for useful suggestions, Aaron Dutton and Michele Cappellari for comments/discussions on stellar IMFs. KHC is grateful for the sabbatical leave (March 2010 - January 2011) at Fermilab Center for Particle Astrophysics where significant parts of this work were carried out. He thanks in particular Albert Stebbins and Craig Hogan at FCPA for their encouragements. He also thanks In-Taek Gong at Sejong University for his assistance in preparing a figure. AVK was supported in part by the 
NSF grant AST-0708154, and by the Kavli Institute for Cosmological Physics at the University of Chicago through the NSF grant PHY-0551142 and PHY-1125897 and an endowment from the Kavli Foundation. MB is grateful for partial support provided by NASA grant ADP/NNX09AD02G.

\section{A SDSS early-type galaxy parameter correlations}

An early-type galaxy with $M_{\star}$ and $\sigma$ specified may be given its stellar mass density profile using observed correlations with $R_{\mathrm{e}}$ (effective radius) and $n$ (Sérsic index) assuming the Sérsic stellar mass density profile [54] given by

$$
\Sigma_{\star}(X)=A_{n} \exp \left(-b_{n} X^{1 / n}\right),
$$

where $X=R / R_{\mathrm{e}}$ ( $R$ being the two dimensional radius), $b_{n}=2 n-1 / 3+0.009876 / n(0.5<$ $n<10)$, and $A_{n}=b_{n}^{2 n} /[2 \pi n \Gamma(2 n)][83,84]$. This is the projected two dimensional density normalized such that its integrated total mass is unity. The deprojected three dimensional density at $x=r / R_{\mathrm{e}}$ (the three dimensional radius in units of $R_{\mathrm{e}}$ ) is then given by

$$
\rho_{\star}(x)=\rho_{n} x^{-\alpha_{n}} \exp \left(-b_{n} x^{1 / n}\right)
$$

with $\alpha_{n}=1-0.6097 / n+0.05563 / n^{2}$ and $\rho_{n}=\left(b_{n}\right)^{n\left(3-\alpha_{n}\right)} /\left\{4 \pi n \Gamma\left[n\left(3-\alpha_{n}\right)\right]\right\}[83,84]$.

To a galaxy with $M_{\star}$ and $\sigma$ specified we give a specific value of $R_{\mathrm{e}}$ using the observed distribution of $M_{\mathrm{dyn}} / M_{\star}$ as a function of $M_{\star}$, where $M_{\mathrm{dyn}} \equiv 5 R_{\mathrm{e}} \sigma^{2} / G$ ( $G$ being Newton's gravitational constant) is a 'dynamical' mass. Fig. 14 shows the observed distribution of $M_{\mathrm{dyn}} / M_{\star}$. Notice that the distribution of $M_{\mathrm{dyn}} / M_{\star}$ essentially shows the scatter of the fundamental mass plane relation of early-type galaxies for three parameters $R_{\mathrm{e}}, \sigma$ and $M_{\star}$.

Finally, we give a specific value of $n$ to a galaxy using the observed distribution of $n$ as a function of $M_{\star}$ (see Fig. 15). This result comes also from SDSS data [42]. Sérsic index $n$ may also be correlated with $\sigma$ and $R_{\mathrm{e}}$. However, the current unavailability of such correlations forces us to use only the correlation shown in Fig. 15.

\section{B An integral solution of the Spherical Jeans equation}

For a specific form of the velocity dispersion anisotropy $\beta(r)$ the radial velocity dispersion $\sigma_{\mathrm{r}}(r)$ can be expressed as an integral form that can be easily evaluated numerically.

We recast the spherical Jeans equation [eq. (4.1)] as

$$
\frac{d y(r)}{d r}+p(r) y(r)=q(r),
$$

where $y(r) \equiv \rho_{\star}(r) \sigma_{\mathrm{r}}^{2}(r), p(r) \equiv 2 \beta(r) / r$, and $q(r) \equiv-G \rho_{\star}(r) M(r) / r^{2}$. Then, with the definition of $\omega(r) \equiv \exp \left[\int^{r} p(t) d t\right]$ we get

$$
y(r)=-\frac{1}{\omega(r)} \int_{r}^{\infty} \omega(t) q(t) d t .
$$

Let us now consider a general functional form for $\beta(r)$ given by

$$
\beta(r)=\beta_{0}+\beta_{1} \frac{r^{2}}{r^{2}+r_{1}^{2}}+\beta_{2} \frac{r^{2}}{r^{2}+r_{2}^{2}},
$$




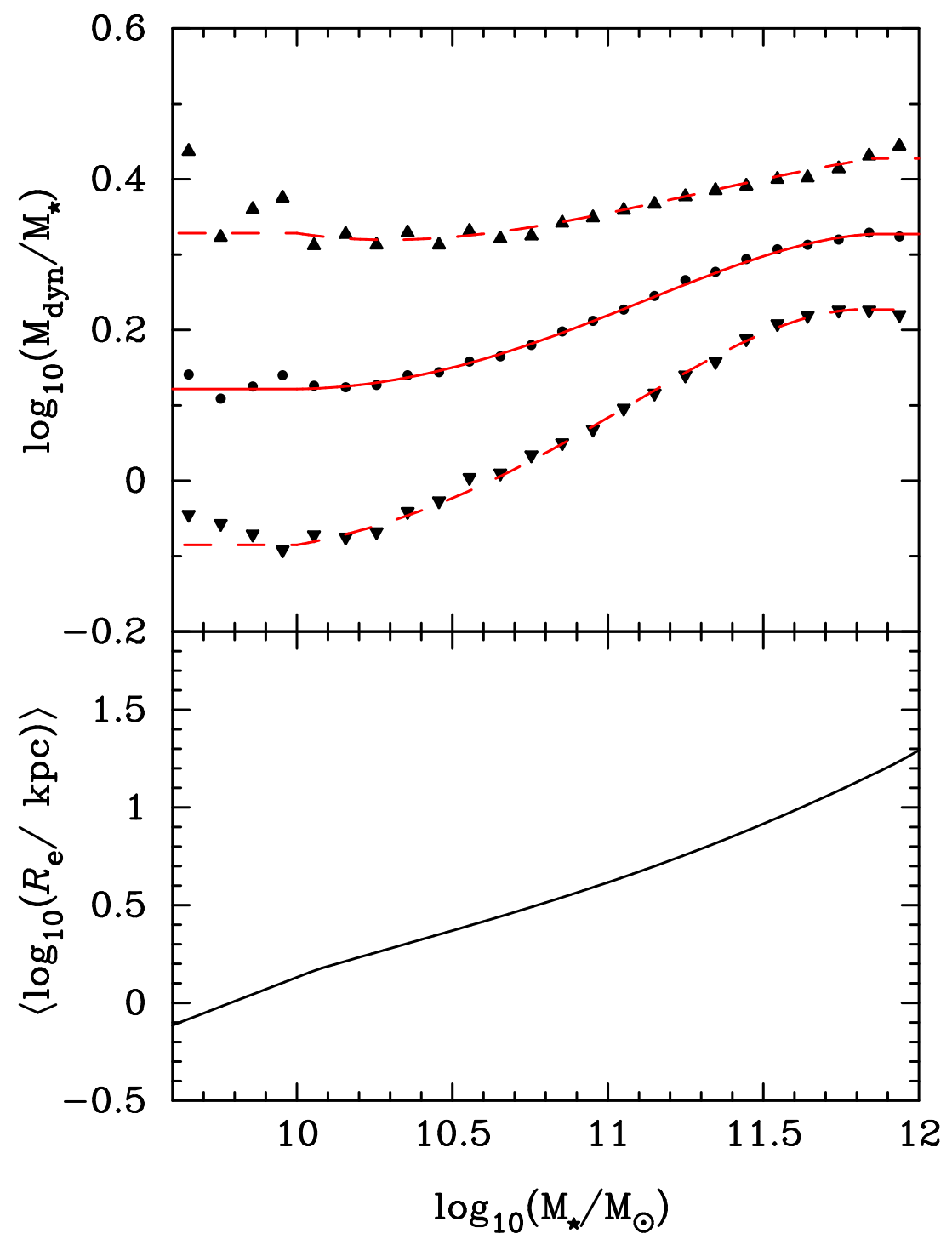

Figure 14. (Top) The distribution of $\log _{10}\left(M_{\mathrm{dyn}} / M_{\star}\right)$ for SDSS early-type galaxies with $M_{\mathrm{dyn}} \equiv$ $5 R_{\mathrm{e}} \sigma^{2} / G$. We obtain this distribution from the data analyzed in [41]. Data points are the median values and $68 \%$ scatters. Red solid curve and dashed curves are the polynomial fits assuming the Gaussian distribution. (Bottom) The distribution of the mean value of $\log _{10}\left(R_{\mathrm{e}} / \mathrm{kpc}\right)$ based on the Gaussian mean of $\log _{10}\left(M_{\mathrm{dyn}} / M_{\star}\right)$ from the top panel and the mean of $\log _{10}\left(\sigma / \mathrm{km} \mathrm{s}^{-1}\right)$ from Fig. 5 .

where $r_{1}<r_{2}$ and $\beta_{0}$ is the anisotropy at the origin. This model allows an extremum anisotropy at a finite, non-zero $r$. We can relate $\beta_{1}$ and $\beta_{2}$ in equation (B.3) to a mean anisotropy within $r_{c}\left(\beta_{\text {mean }}\right)$ and the anisotropy at infinity $\left(\beta_{\infty}\right)$ as follows:

$$
\begin{aligned}
& \beta_{1}=\frac{\beta_{\text {mean }}-\beta_{0}\left(1-u_{2}\right)-\beta_{\infty} u_{2}}{u_{1}-u_{2}}, \\
& \beta_{2}=\beta_{\infty}-\beta_{0}-\beta_{1},
\end{aligned}
$$

where $u_{1}=1-\left(r_{1} / r_{c}\right) \arctan \left(r_{c} / r_{1}\right)$ and $u_{2}=1-\left(r_{2} / r_{c}\right) \arctan \left(r_{c} / r_{2}\right)$. Fig. 16 shows 


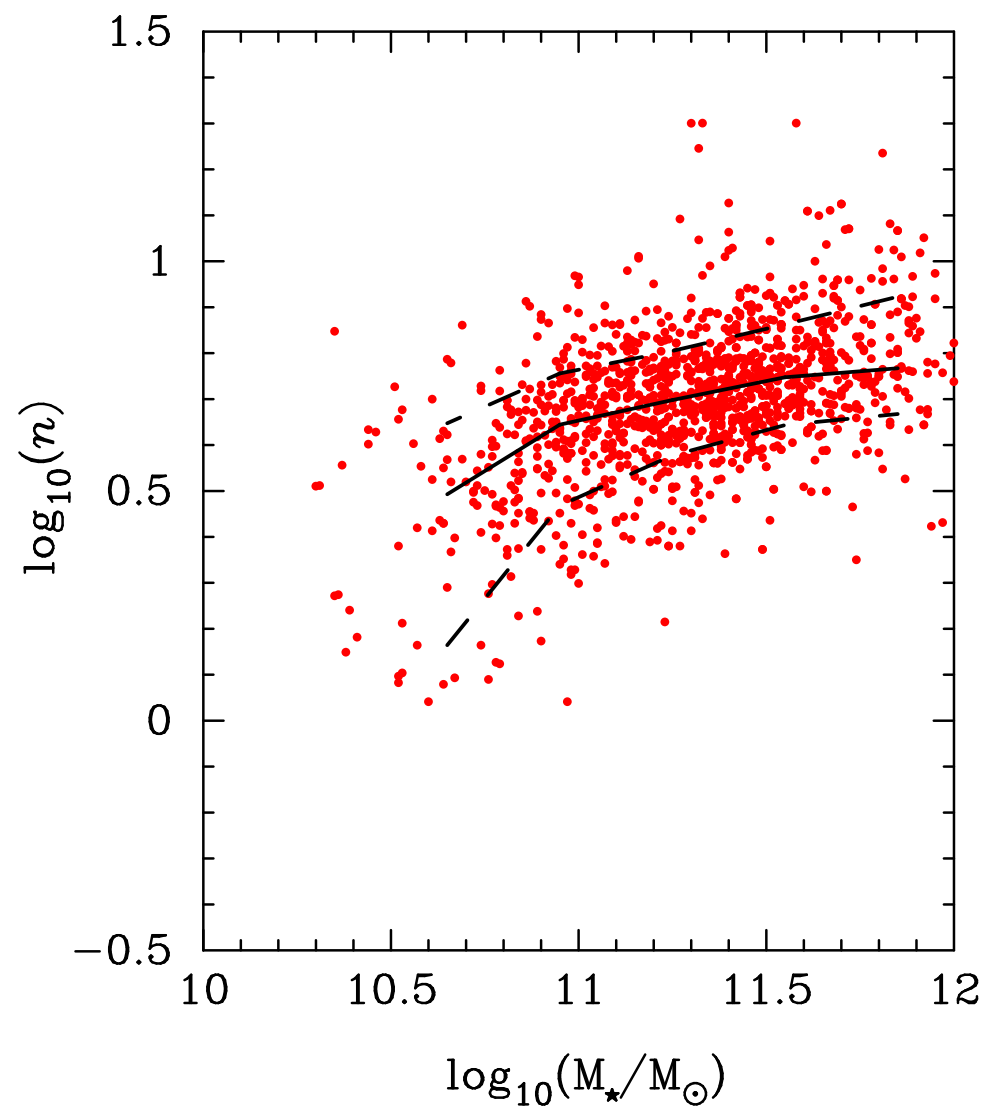

Figure 15. The distribution of Sérsic index $(n)$ as a function of stellar mass $\left(M_{\star}\right)$ for SDSS earlytype galaxies (red points) with the curves showing median values (full) and $68 \%$ scatters (dashed). These data are taken from [42].

examples of varying anisotropies based on equation (B3) for which we consider three cases of $\beta_{\text {mean }}\left(r<R_{\mathrm{e}}\right)=0,+0.3,-0.3$ and take values from the ranges $-0.3<\beta_{0}<0.3,-0.9<$ $\beta_{\infty}<0.5,0<r_{1}<R_{\mathrm{e}}$ and $0<r_{2}<R_{\mathrm{e}}$ (with $r_{2}>r_{1}$ ).

For this model $\omega(r)$ becomes

$$
\omega(r)=r^{2 \beta_{0}}\left(r^{2}+r_{1}^{2}\right)^{\beta_{1}}\left(r^{2}+r_{1}^{2}\right)^{\beta_{2}} .
$$

Substituting this into equation (B.2) we get

$$
\sigma_{\mathrm{r}}^{2}(r)=G \int_{r}^{\infty} \frac{\omega(t)}{\omega(r)} \frac{\rho_{\star}(t)}{\rho_{\star}(r)} \frac{M(t)}{t^{2}} d t
$$

For the case of constant anisotropy $\beta=\beta_{0}=\beta_{\text {mean }}=\beta_{\infty}$ it takes the following simple form

$$
\sigma_{\mathrm{r}}^{2}(r)=G \frac{r^{-2 \beta}}{\rho_{\star}(r)} \int_{r}^{\infty} t^{2(\beta-1)} \rho_{\star}(t) M(t) d t .
$$



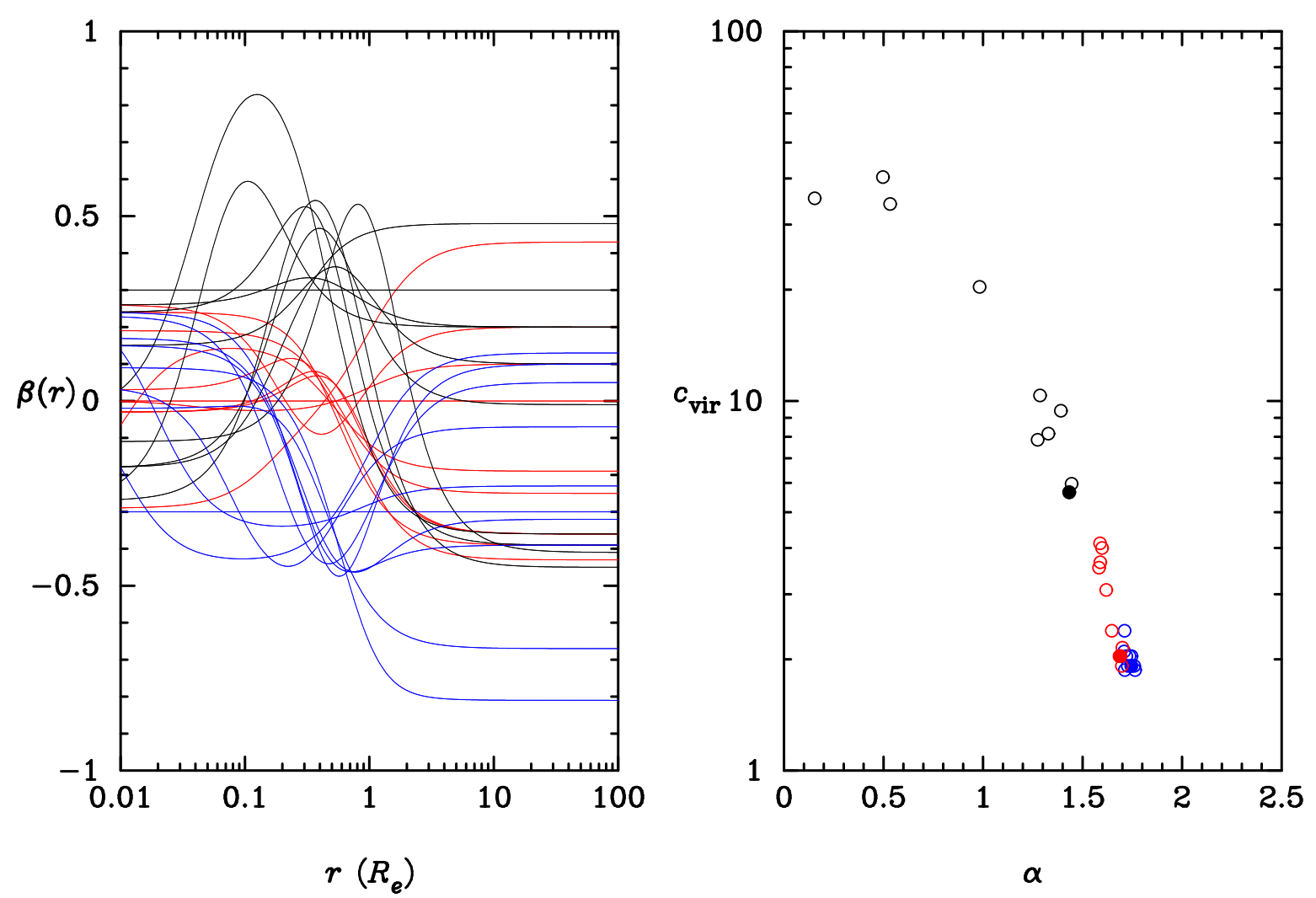

Figure 16. (Left) Examples of varying anisotropy $\beta(r)$ based on the model given by equation (B3) that solve for the spherical Jeans equation for a system with $M_{\text {vir }}=10^{13} \mathrm{M}_{\odot}$ (see the text). Black, red and blues curves are respectively for $\beta_{\text {mean }}=+0.3,0,-0.3$. (Right) Dark matter density profile parameters of the $\alpha \mathrm{NFW}$ model (eq. 4.6) matching the anisotropies shown left for the same system. For $\beta_{\text {mean }}=+0.3$ varying anisotropies (open black circles) can give significantly different values of $\alpha$ and $c_{\text {vir }}$ compared with the case of constant anisotropy (filled black circle) whereas for $\beta_{\text {mean }}=-0.3$ (blue) varying anisotropies have little effects on $\alpha$ or $c_{\text {vir }}$. For $\beta_{\text {mean }}=0$ (red), varying anisotropies can change appreciably $c_{\text {vir }}$ only.

In the above the total mass within $r$ is given by $M(r)=M_{\star}(r)+M_{\mathrm{dm}}(r)$ with the stellar mass

$$
M_{\star}(r)=M_{\star} \frac{\gamma\left[n\left(3-\alpha_{n}\right), b_{n}\left(r / R_{\mathrm{e}}\right)^{1 / n}\right]}{\Gamma\left[n\left(3-\alpha_{n}\right)\right]}
$$

and the dark matter mass

$$
M_{\mathrm{dm}}(r)=\left(M_{\mathrm{vir}}-M_{\star}\right) \frac{f_{\alpha}\left(r / r_{s}\right)}{f_{\alpha}\left(c_{\mathrm{vir}}\right)}
$$

for the $\alpha \mathrm{NFW}$ model and

$$
M_{\mathrm{dm}}(r)=\left(M_{\mathrm{vir}}-M_{\star}\right) \frac{\tilde{f}_{\tilde{\alpha}}\left(r / r_{-2}\right)}{\tilde{f}_{\tilde{\alpha}}\left(\tilde{c}_{\mathrm{vir}}\right)},
$$

for the Einasto model. In the above $\Gamma(x)$ and $\gamma(x, y)$ are the gamma function and the incomplete gamma function respectively. The functions $f_{\alpha}(x)$ and $\tilde{f}_{\tilde{\alpha}}(x)$ are given by equation (4.9) and equation (4.13) respectively. 


\section{Effects of varying anisotropies of velocity dispersions}

In the main text we assume that anisotropies of velocity dispersions are constant in radius. Constant anisotropies are used mainly because of the computational simplicity and in part because of the lack of empirical statistical characterization of radial behaviors of anisotropies. Here we consider artificial varying anisotropies $\beta(r)$ using equation (B.3) introduced above to test whether the constancy of anisotropies is likely to have biased our results on dark matter density profiles.

Both observed anisotropies of stellar kinematics [6] and simulated anisotropies of dark matter kinematics [63] show that real anisotropies can vary significantly with $r$ in the inner region of halos. However, those anisotropies are bounded within $-0.9 \lesssim \beta(r) \lesssim 0.5$ for all probed $r$. In particular, $\beta(r) \sim 0$ as $r \rightarrow 0$. Using equation (B.3) for $\beta(r)$ we modify the procedure of the main text (section 4.3) as follows. For each galaxy we fix $\beta_{\text {mean }}$ within $R_{\mathrm{e}}$ using a value $x$ drawn randomly from a probability density function

$$
P(x) \propto[1-\theta(x-\mu)] \exp \left[-\frac{(x-\mu)^{2}}{2 \sigma_{\mathrm{L}}^{2}}\right]+\theta(x-\mu) \exp \left[-\frac{(x-\mu)^{2}}{2 \sigma_{\mathrm{H}}^{2}}\right]
$$

with $\mu=0.18, \sigma_{\mathrm{H}}=0.11$ and $\sigma_{\mathrm{L}}=0.25$ based on $\sim 40$ unoverlapping early-type galaxies in the literature $[6,66]$. This rather strong prior is necessary because of the great freedom allowed by equation (B.3) and is justified because it comes from observed galaxies. We then vary the rest of the anisotropy parameters $\left(\beta_{0}, \beta_{\infty}, r_{1}\right.$, and $\left.r_{2}\right)$ to create a degenerate model set for each system using the following priors: $-0.3<\beta_{0}<0.3$, equation (4.14) for $\beta_{\infty}$, $0<r_{1}<R_{\mathrm{e}}$, and $0<r_{2}<R_{\mathrm{e}}$ with $r_{1}<r_{2}$ (this range for $r_{1}$ and $r_{2}$ is chosen to allow for rapidly varying in the inner region). These ranges are intended to encompass observed variations in early-type galaxies. Fig. 17 shows the results on the dark matter density profiles based on the Chabrier stellar masses matching Fig. 10 with constant anisotropies. We notice that the statistical distributions of the parameters are quite similar to those for the case of constant anisotropy, with only small offsets of the means between the two cases.

To understand the above results we make a detailed case study of a system with $M_{\text {vir }}=$ $10^{13} \mathrm{M}_{\odot}, M_{\star}=10^{11.13} \mathrm{M}_{\odot}, \sigma=10^{2.308} \mathrm{~km} \mathrm{~s}^{-1}, R_{\mathrm{e}}=4.86 \mathrm{kpc}$ and $n=4.74$. Assuming that the velocity dispersion profile slope at $R_{\mathrm{e}} / 2$ is $\eta=-0.053 \pm 0.044$, we try various anisotropies to see how the resulting dark matter density profile depends on anisotropy. We pick three cases of $\beta_{\text {mean }}=+0.3,0,-0.3$ within $R_{\mathrm{e}}$. For each value of $\beta_{\text {mean }}$ we try ten different shapes of the anisotropy, one constant case and nine other varying cases randomly selected from the ranges specified above that satisfy the Jeans equation and $\eta=-0.053$ up to the error of 0.044. Fig. 16 shows a total of 30 anisotropies and the corresponding dark matter profiles based on the $\alpha \mathrm{NFW}$ model (eq. 4.6). First of all, comparing three constant cases we find that the resulting dark matter density profile varies systematically as a function of $\beta_{\text {mean }}$; $\alpha\left(c_{\mathrm{vir}}\right)$ decreases (increases) with $\beta_{\text {mean }}$. Secondly, for the case of $\beta_{\text {mean }}=-0.3$ (colored blue) varying anisotropies have little effects on $\alpha$ and $c_{\mathrm{vir}}$. Thirdly, for zero mean anisotropy $\beta_{\text {mean }}=0$ (colored red) varying shapes give on average significantly larger $c_{\mathrm{vir}}$ and a little lower $\alpha$ compared with the constant case. Finally, for the case of $\beta_{\text {mean }}=+0.3$ (colored black) varying shapes can change $\alpha$ and $c_{\text {vir }}$ significantly (in some cases dramatically). Interestingly, the direction of movement in the parameter space due to varying anisotropy shapes from a constant is the same as that due to increasing $\beta_{\text {mean }}$. This means that the effect of varying shape can be mimicked by increasing $\beta_{\text {mean }}$.

As shown in the above case study a varying anisotropy can lead to a significant change in the resulting dark matter density profile of an individual system. Hence, if we kept the 

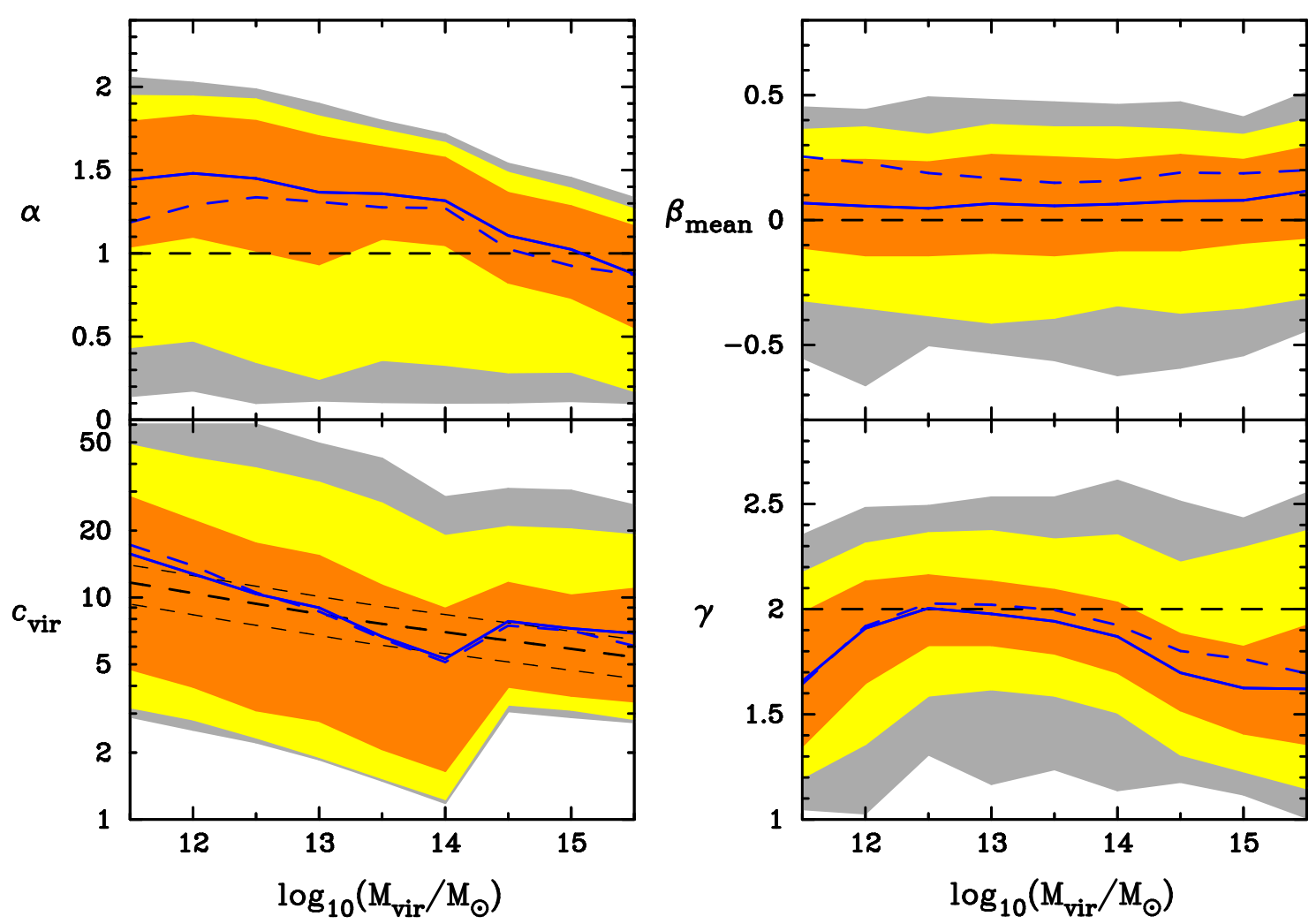

Figure 17. Same as Fig. 10 except that this result is based on varying anisotropies given by equation (B.3). Blue full and dashed curves show respectively the expectation values for the cases of varying and constant anisotropies. Notice that there are only small offsets between the two.

population mean of $\beta_{\text {mean }}$ fixed, the statistical properties of resulting dark matter density profiles would depend on whether we use constant or varying anisotropies. However, in our procedure with constant anisotropy (section 4.3.2) we allowed $\beta_{\text {mean }}$ to take values stochastically from a prior range, whereas in the procedure with varying anisotropy used here we allowed shapes to vary stochastically within prior ranges while imposing a prior empirical distribution of $\beta_{\text {mean }}$. Because the effect of a varying anisotropy can be mimicked by changing $\beta_{\text {mean }}$, it turns out that the statistical properties of dark matter density profiles are similar between the two procedures with a (small) offset in $\beta_{\text {mean }}$. This indicates that according to our stochastic procedure the resulting distribution of dark matter density profiles is minimally dependent on anisotropy models. Therefore, unless true anisotropy shapes and/or the true mean of $\beta_{\text {mean }}$ are significantly different from those adopted here, it is unlikely that our assumption of constant anisotropy has significantly biased our results on dark matter density profiles. 


\section{Dynamical mass scaling relations and alternative results for halo mass profile}

Our results on the halo profiles presented in the main text (section 4.3.2) are based on the observational constraints on the velocity dispersion profile (VP). Dynamical mass scaling relations have been presented recently in the literature [72, 73]. These relations are derived from the spherical Jeans equation in conjunction with physically well-motivated dynamical assumptions that are consistent with current observations. These relations allow us to estimate dynamical masses within certain radii from velocity dispersions. A crucial common feature of these relations is that they are insensitive to the value of the velocity dispersion anisotropy $\beta$.

The first mass estimate [72] is expressed as

$$
M^{(\text {est })}\left(r_{\mathrm{opt}}\right)=\frac{f_{v}^{-2}}{G} r_{\mathrm{opt}} \sigma_{\text {los }}^{2}\left(r_{\mathrm{opt}}\right),
$$

where $r_{\mathrm{opt}}$ is an optimal radius defined in the reference and $r_{\mathrm{opt}} \sim 0.5 R_{\mathrm{e}}$ typically. The numerical factor $f_{v}$ is a velocity ratio defined in the reference and typically $f_{v} \sim 0.6$. This mass estimate is based on the assumption that the total gravitational potential is that of the isothermal potential. This is not strictly satisfied by observed galaxies but a reasonable approximation for the potential within stellar extents of galaxies supported by various astrophysical studies including strong lensing [9-11].

The second mass estimate [73] is expressed as

$$
M^{(\mathrm{est})}\left(r_{1 / 2}\right)=\frac{3}{G} r_{1 / 2}\left\langle\sigma_{\text {los }}^{2}\right\rangle(\infty),
$$

where $r_{1 / 2}$ is the three-dimensional radius at which the enclosed stellar mass is $M_{\star} / 2$ and $r_{1 / 2} \approx 4 R_{\mathrm{e}} / 3$ for most stellar mass distributions of spheroids. This mass estimate is based on the assumption that $\sigma_{\text {los }}(R)$ varies sufficiently slowly with $R$ near $R_{\mathrm{e}}$ as supported by current observations [66, 71].

Because of the approximate assumptions made we expect that our galaxy-halo systems will satisfy the above mass scaling relations only approximately. Fig. 18 shows the ratios of the estimated masses based on the scaling relations to the masses enclosed within the relevant radii for our systems. For $10^{12} \mathrm{M}_{\odot}<M_{\text {vir }}<10^{14} \mathrm{M}_{\odot}$ the mean estimated masses agree with the mean enclosed masses within $10 \%$. However, the mean ratio $\left\langle M^{(\mathrm{est})}\left(r_{\mathrm{opt}}\right) / M\left(r_{\mathrm{opt}}\right)\right\rangle$ (solid curves in the left panel of Fig. 18) tends to lie above unity whereas the mean ratio $\left\langle M^{\text {(est) }}\left(r_{1 / 2}\right) / M\left(r_{1 / 2}\right)\right\rangle$ (solid curves in the right panel of Fig. 18) systematically varies as a function of $M_{\mathrm{vir}}$. We do not speculate on possible sources of these behaviors.

In the above we have tested the dynamical mass scaling relations with our systems constrained by the VP constraints only. Alternatively, we could use the dynamical mass scaling relations as additional constraints on the halo dark matter density profiles. We proceed by introducing two figure-of-merit functions defined by

$$
Q_{(\mathrm{Chur})}^{2} \equiv \frac{1}{\delta^{2}}\left(\frac{M^{\mathrm{est}}\left(r_{\mathrm{opt}}\right)-M\left(r_{\mathrm{opt}}\right)}{M\left(r_{\mathrm{opt}}\right)}\right)^{2}
$$

and

$$
Q_{(\text {Wolf })}^{2} \equiv \frac{1}{\delta^{2}}\left(\frac{M^{\mathrm{est}}\left(r_{1 / 2}\right)-M\left(r_{1 / 2}\right)}{M\left(r_{1 / 2}\right)}\right)^{2}
$$




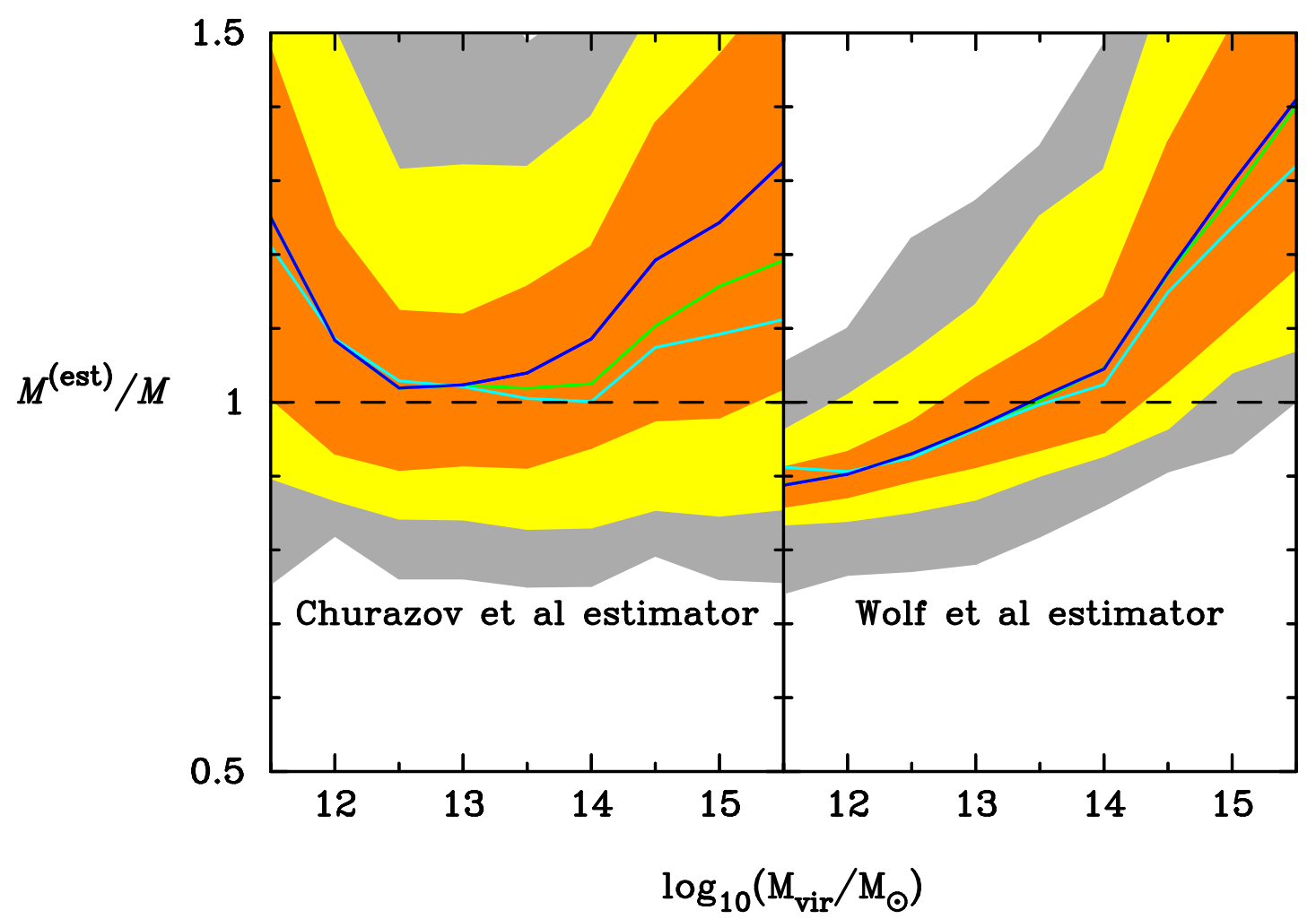

Figure 18. Ratios of the estimated masses based on two scaling relations from the literature to the masses within two radii $r_{1 / 2}\left(\approx 4 R_{\mathrm{e}} / 3\right.$, left panel $)$ and $r_{\text {opt }}\left(\sim R_{\mathrm{e}} / 2\right.$, right panel $)$ for our galaxy-halo systems based on the observational VP constraints. The orange, yellow and gray regions include the $68 \%, 95 \%$ and $99.7 \%$ of our systems respectively. The blue curves are the average values. The green and cyan curves are for the corrected stellar masses as in Fig. 10.

where we assume $\delta=0.1$. We then combine these functions to the function $Q_{(\mathrm{VP})}^{2}$ based on the VP constraints given by equation (4.16) with $x$ replaced by $\langle\eta\rangle$. In doing so we perform a sort of least-square fitting based on two independent constraints. Fig. 19 and Fig. 20 show the results on the $\alpha \mathrm{NFW}$ model based on combined figure-of-merit functions given by $Q_{(\mathrm{VP})}^{2}+Q_{(\mathrm{Chur})}^{2}$ and $Q_{(\mathrm{VP})}^{2}+Q_{(\text {Wolf })}^{2}$. Fig. 19 is quite similar to Fig. 10 except for $M_{\text {vir }} \lesssim 10^{12} \mathrm{M}_{\odot}$. Fig. 20 shows some quantitative difference with Fig. 10 in the behaviors of $\alpha$ and $c_{\mathrm{vir}}$.

\section{References}

[1] S. D. M. White and M. J. Rees, Core condensation in heavy halos - A two-stage theory for galaxy formation and clustering, Mon. Not. R. Astron. Soc. 183 (1978) 341

[2] S. D. M. White and C. S. Frenk, Galaxy formation through hierarchical clustering, Astrophys. J. 379 (1991) 52

[3] V. C. Rubin, W. K. Ford Jr. and N. Thonnard, Rotational properties of 21 SC galaxies with a 

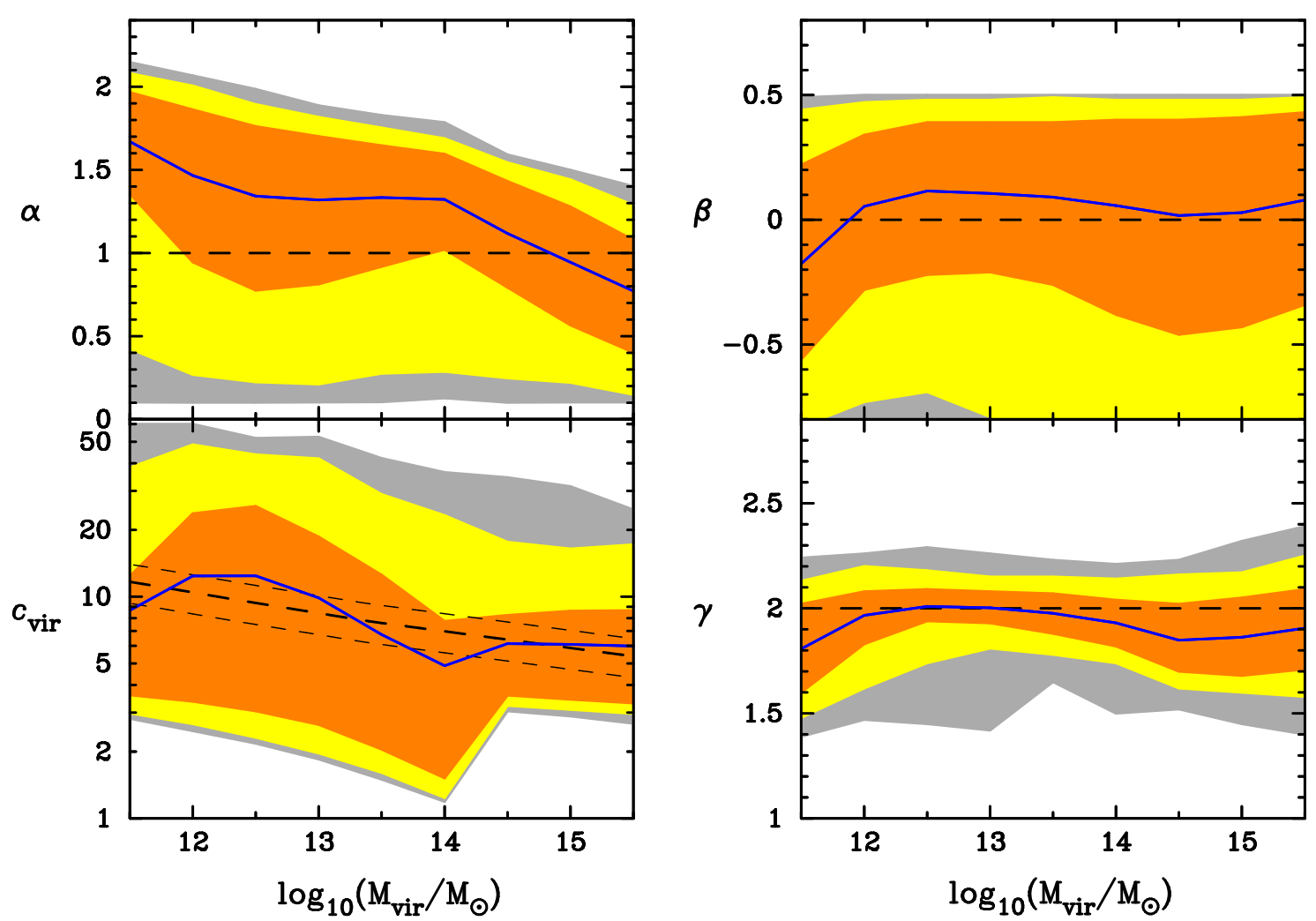

Figure 19. Same as Fig. 10 except that this result is based on the combined figure-of-merit function of $Q_{(\mathrm{VP})}^{2}+Q_{(\mathrm{Chur})}^{2}$ [equation (4.14) \& equation (C.3)].

large range of luminosities and radii, from NGC $4605 R=4 k p c$ to $U G C 2885 R=122 \mathrm{kpc}$, Astrophys. J. 238 (1980) 471

[4] S. M. Kent, Dark matter in spiral galaxies. I - Galaxies with optical rotation curves, Astron. J. 91 (1986) 1301

[5] M. Persic, P. Salucci and F. Stel, The universal rotation curve of spiral galaxies - I. The dark matter connection, Mon. Not. R. Astron. Soc. 281 (1996) 27

[6] O. Gerhard, A. Kronawitter, R. P. Saglia and R. Bender, Dynamical family properties and dark halo scaling relations of giant elliptical galaxies, Astron. J. 121 (2001) 1936

[7] A. Dekel, F. Stoehr, G. A. Mamon, T. J. Cox, G. S. Novak and J. R. Primack, Lost and found dark matter in elliptical galaxies, Nature 437 (2005) 707

[8] M. Cappellari et al., The SAURON project - IV. The mass-to-light ratio, the virial mass estimator and the Fundamental Plane of elliptical and lenticular galaxies, Mon. Not. R. Astron. Soc. 366 (2006) 1126

[9] D. Rusin, C. S. Kochanek and C. R. Keeton, Self-similar models for the mass profiles of early-type lens galaxies, Astrophys. J. 595 (2003) 29

[10] L. V. E. Koopmans, A. Bolton, T. Treu, O. Czoske, M. W. Auger, M. Barnabè, S. Vegetti, R. Gavazzi, L. A. Moustakas and S. Burles, The structure and dynamics of massive early-type galaxies: On homology, isothermality, and isotropy inside one effective radius, Astrophys. J. 703 

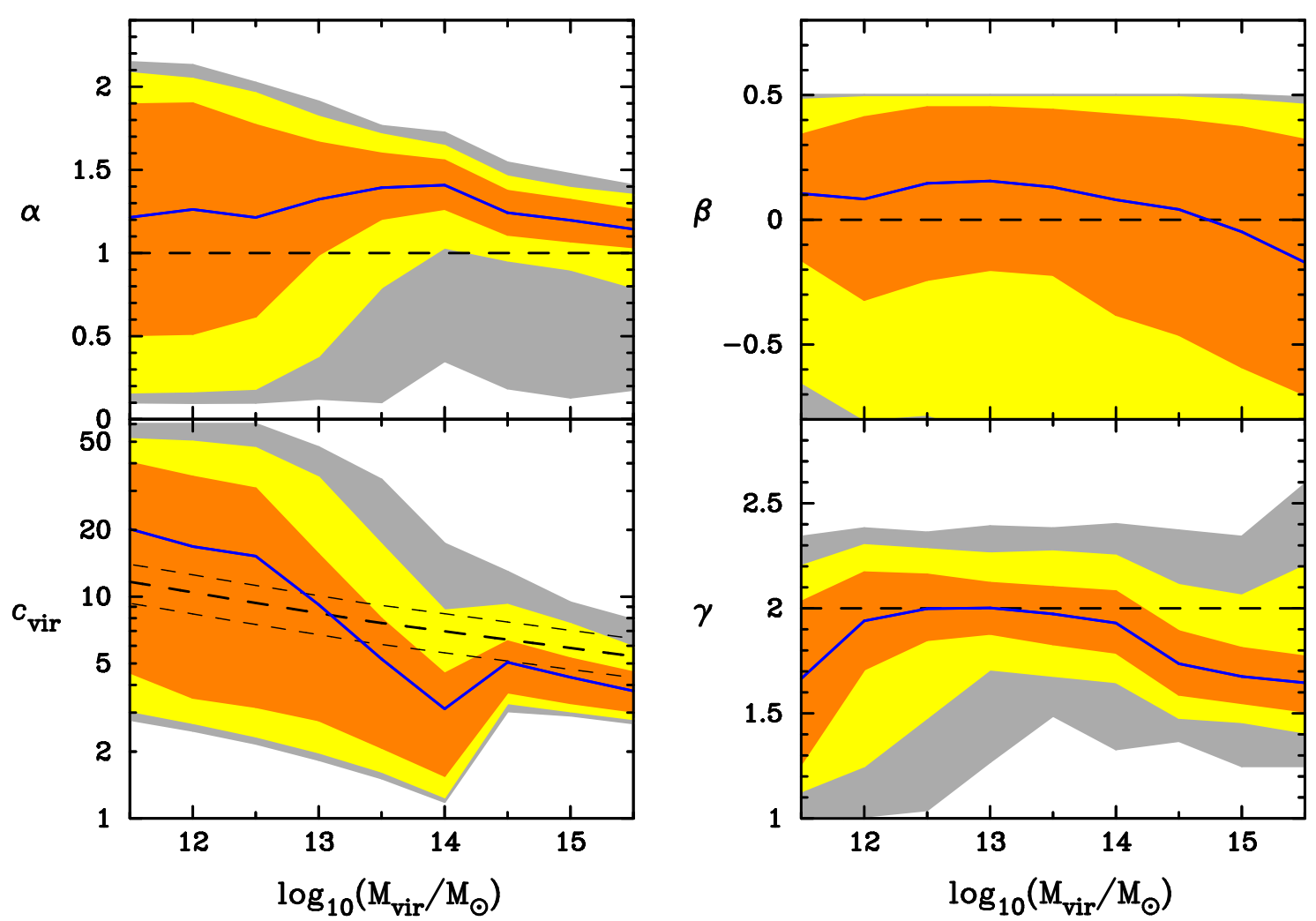

Figure 20. Same as Fig. 10 except that this result is based on the combined figure-of-merit function of $Q_{(\mathrm{VP})}^{2}+Q_{(\text {Wolf })}^{2}$ [equation (4.14) \& equation (C.4)].

(2009) L51

[11] M. Barnabè, O. Czoske, L. V. E. Koopmans, T. Treu and A. S. Bolton, Two-dimensional kinematics of SLACS lenses - III. Mass structure and dynamics of early-type lens galaxies beyond $z \simeq$ 0.1, Mon. Not. R. Astron. Soc. 415 (2011) 2215

[12] D. Clowe, M. Bradac, A. H. Gonzalez, M. Markevitch, S. W. Randall, C. Jones and D. Zaritsky, A direct empirical proof of the existence of dark matter, Astrophys. J. 648L (2006) 109

[13] J.-P. Kneib, P. Hudelot, R. S. Ellis, T. Treu, G. P. Smith, P. Marshall, O. Czoske, I. Smail and P. Natarajan, A wide-field Hubble Space Telescope study of the cluster Cl 0024+1654 at $z=0.4$. II. The cluster mass distribution, Astrophys. J. 598 (2003) 804

[14] V. Springel et al., Simulations of the formation, evolution and clustering of galaxies and quasars, Nature 435 (2005) 629

[15] C. Conroy, R. H. Wechsler and A. V. Kravtsov, Modeling luminosity-dependent galaxy clustering through cosmic time, Astrophys. J. 647 (2006) 201

[16] I. Zehavi et al., Galaxy clustering in the completed SDSS redshift survey: the dependence on color and luminosity, Astrophys. J. 736 (2011) 59

[17] G. Bertone, D. Hooper and J. Silk, Particle dark matter: evidence, candidates and constraints, Phys. Rep. 405 (2005) 279

[18] J. L. Feng, Dark matter candidates from particle physics and methods of detection, Ann. Rev. 
Astron. Astrophys. 48 (2010) 495

[19] T. A. Porter, R. P. Johnson and P. W. Graham, Dark matter searches with astroparticle data, Ann. Rev. Astron. Astrophys. 49 (2011) 155

[20] A. E. Schulz, R. Mandelbaum and N. Padmanabhan, Testing adiabatic contraction with Sloan Digital Sky Survey elliptical galaxies, Mon. Not. R. Astron. Soc., 408, 1463 (2010)

[21] R. Reyes, R. Mandelbaum, J. E. Gunn, R. Nakajima, U. Seljak and C. M. Hirata, Virial-to-optical velocity ratios of local disk galaxies from combined kinematics and galaxy-galaxy lensing, Mon. Not. R. Astron. Soc. submitted (arXiv:1110.4107)

[22] M. W. Auger, T. Treu, R. Gavazzi, A. S. Bolton, L. V. E. Koopmans and P. J. Marshall, Dark matter contraction and the stellar content of massive early-type galaxies: disfavoring "light" initial mass functions, Astrophys. J. 721L (2010) 163

[23] M. Oguri, M. B. Bayliss, H. Dahle, K. Sharon, M. D. Gladders, P. Natarajan, J. F. Hennawi and B. P. Koester, Combined strong and weak lensing analysis of 28 clusters from the Sloan Giant Arcs Survey, Mon. Not. R. Astron. Soc. in press (arXiv:1109.2594v2)

[24] O. Y. Gnedin, D. Ceverino, N. Y. Gnedin, A. A. Klypin, A. V. Kravtsov, R. Levine,D. Nagai and G. Yepes, Halo contraction effect in hydrodynamic simulations of galaxy formation, Astrophys. J. submitted (arXiv:1108.5736)

[25] A. R. Duffy, J. Schaye, S. T. Kay, C. Dalla Vecchia, R. A. Battye and C. M. Booth, Impact of baryon physics on dark matter structures: a detailed simulation study of halo density profiles, Mon. Not. R. Astron. Soc. 405 (2010) 2161

[26] M. G. Abadi, J. F. Navarro, M. Fardal, A. Babul and M. Steinmetz, Galaxy-induced transformation of dark matter haloes, Mon. Not. R. Astron. Soc. 407 (2010) 435

[27] P. B. Tissera, S. D. M. White, S. Pedrosa and C. Scannapieco, Dark matter response to galaxy formation, Mon. Not. R. Astron. Soc. 406 (2010) 922

[28] P. H. Johansson, T. Naab and J. P. Ostriker, Gravitational heating helps make massive galaxies red and dead, Astrophys. J. 697L (2009) 38

[29] S. Trujillo-Gomez, A. Klypin, J. Primack and A. J. Romanowsky, Galaxies in $\Lambda$ CDM with halo abundance matching: luminosity-velocity relation, baryonic mass-velocity relation, velocity function, and clustering, Astrophys. J. 742 (2011) 16

[30] A. A. Dutton, C. Conroy, F. C. van den Bosch, L. Simard, J. T. Mendel, S. Courteau, A. Dekel, S. More and F. Prada, Dark halo response and the stellar initial mass function in early-type and late-type galaxies, Mon. Not. R. Astron. Soc. 416 (2011) 322

[31] A. J. Benson and R. Bower, Galaxy formation spanning cosmic history, Mon. Not. R. Astron. Soc. 405 (2010) 1573

[32] X. X. Xue et al., The Milky Way's circular velocity curve to $60 \mathrm{kpc}$ and an estimate of the dark matter halo mass from the kinematics of 2400 SDSS blue horizontal-branch stars, Astrophys. J. 684 (2008) 1143

[33] M. S. Seigar, A. J. Barth and J. S. Bullock, A revised $\Lambda$ CDM mass model for the Andromeda Galaxy, Mon. Not. R. Astron. Soc. 389 (2008) 1911

[34] A. Sonnenfeld, T. Treu, R. Gavazzi, P. J. Marshall, M. W. Auger, S. H. Suyu, L. V. E. Koopmans and A. S. Bolton, Evidence for dark matter contraction and a Salpeter IMF in a massive early-type galaxy, Astrophys. J. submitted (arXiv:1111.4215)

[35] N. R. Napolitano et al., The PN.S Elliptical Galaxy Survey: a standard $\Lambda$ CDM halo around NGC 4374?, Mon. Not. R. Astron. Soc. 411 (2011) 2035

[36] K. Umetsu, T. Broadhurst, A. Zitrin, E. Medezinski, D. Coe and M. Postman, A precise cluster mass profile averaged from the highest-quality lensing data, Astrophys. J. 738 (2011) 41 
[37] A. B. Newman, T. Treu, R. S. Ellis and D. J. Sand, The dark matter distribution in A383: evidence for a shallow density cusp from improved lensing, stellar kinematic, and X-ray data, Astrophys. J. 728L (2011) 39

[38] C. McCabe, Astrophysical uncertainties of dark matter direct detection experiments, Phys. Rev. D 82 (2010) 023530

[39] G. R. Blumenthal, S. M. Faber, R. Flores and J. R. Primack, Contraction of dark matter galactic halos due to baryonic infall, Astrophys. J. 301 (1986) 27

[40] O. Y. Gnedin, A. V. Kravtsov, A. A. Klypin and D. Nagai, Response of dark matter halos to condensation of baryons: cosmological simulations and improved adiabatic contraction model, Astrophys. J. 616 (2004) 16

[41] M. Bernardi, F. Shankar, J. B. Hyde, S. Mei, F. Marulli and R. K. Sheth, Galaxy luminosities, stellar masses, sizes, velocity dispersions as a function of morphological type, Mon. Not. $R$. Astron. Soc. 404 (2010) 2087

[42] Y. Guo et al., Structural properties of central galaxies in groups and clusters, Mon. Not. R. Astron. Soc. 398 (2009) 1129

[43] K. N. Abazajian et al., The Seventh Data Release of the Sloan Digital Sky Survey, Astrophys. J. Suppl. 182 (2009) 543

[44] A. A. Klypin, S. Trujillo-Gomez and J. Primack, Dark matter halos in the standard cosmological model: results from the bolshoi simulation, Astrophys. J. 740 (2011) 102

[45] A. V. Kravtsov, A. A. Berlind, R. H. Wechsler, A. A. Klypin, S. Gottlöber, B. Allgood and J. R. Primack, The dark side of the halo occupation distribution, Astrophys. J. 609 (2004) 35

[46] A. Tasitsiomi, A. V. Kravtsov, R. H. Wechsler and J. R. Primack, Modeling Galaxy-Mass Correlations in Dissipationless Simulations, Astrophys. J. 614 (2004) 533

[47] A. Vale and J. P. Ostriker, Linking halo mass to galaxy luminosity, Mon. Not. R. Astron. Soc. 353 (2004) 189

[48] B. P. Moster, R. S. Somerville, C. Maulbetsch, F. C. van den Bosch, A. V. Macciò, T. Naab and L. Oser, Constraints on the relationship between stellar mass and halo mass at low and high redshift, Astrophys. J. $\mathbf{7 1 0}$ (2010) 903

[49] P. S. Behroozi, C. Conroy and R. H. Wechsler, A comprehensive analysis of uncertainties affecting the stellar mass-halo mass relation for $0 ; z ; 4$, Astrophys. J. 717 (2010) 379

[50] K.-H. Chae, The coevolution of the velocity and mass functions of galaxies and dark haloes, Mon. Not. R. Astron. Soc. 413 (2011) 887

[51] E. Komatsu et al., Seven-year Wilkinson Microwave Anisotropy Probe (WMAP) observations: cosmological interpretation, Astrophys. J. Suppl. 192 (2011) 18

[52] G. L. Bryan and M. L. Norman, Statistical properties of X-Ray clusters: analytic and numerical comparisons, Astrophys. J. 495 (1998) 80

[53] F. Prada, A. A. Klypin, A. J. Cuesta, J. E. Betancort-Rijo and J. Primack, Halo concentrations in the standard LCDM cosmology, Mon. Not. R. Astron. Soc. submitted (arXiv:1104.5130). http://www.multidark.org/MultiDark/

[54] J. L. Sérsic, Atlas de Galaxias Australes (Córdoba: Observatorio Astronómico, 1968)

[55] J. B. Hyde and M. Bernardi, Curvature in the scaling relations of early-type galaxies, Mon. Not. R. Astron. Soc. 394 (2009) 1978

[56] S. More, F. C. van den Bosch, M. Cacciato, R. Skibba, H. J. Mo, X. Yang, Satellite kinematics - III. Halo masses of central galaxies in SDSS, Mon. Not. R. Astron. Soc. 410 (2011) 210

[57] S. More, F. C. van den Bosch, M. Cacciato, H. J. Mo, X. Yang and R. Li, Satellite kinematics - 
II. The halo mass-luminosity relation of central galaxies in SDSS, Mon. Not. R. Astron. Soc. 392 (2009) 801

[58] X. Yang, H. J. Mo and F. C. van den Bosch, Galaxy groups in the SDSS DR4. III. The luminosity and stellar mass functions, Astrophys. J. 695 (2009) 900

[59] R. Mandelbaum, U. Seljak, G. Kauffmann, C. M. Hirata and J. Brinkmann, Galaxy halo masses and satellite fractions from galaxy-galaxy lensing in the Sloan Digital Sky Survey: stellar mass, luminosity, morphology and environment dependencies, Mon. Not. R. Astron. Soc. 368 (2006) 715

[60] F. Shankar, A. Lapi, P. Salucci, G. De Zotti and L. Danese, New relationships between galaxy properties and host halo mass, and the role of feedbacks in galaxy formation, Astrophys. J. 643 (2006) 14

[61] J. Binney, S. Tremaine, Galactic Dynamics, 2nd ed. (Princeton Univ. Press, Princeton, NJ, 2008).

[62] J. F. Navarro, C. S. Frenk and S. D. M. White, A universal density profile from hierarchical clustering, Astrophys. J. 490 (1997) 493

[63] J. F. Navarro, A. Ludlow, V. Springel, J. Wang, M. Vogelsberger, S. D. M. White, A. Jenkins, C. S. Frenk and A. Helmi, The diversity and similarity of simulated cold dark matter haloes, Mon. Not. R. Astron. Soc. 402 (2010) 21

[64] D. Merritt, A. W. Graham, B. Moore, J. Diemand and B. Terzić, Empirical models for dark matter halos. I. Nonparametric construction of density profiles and comparison with parametric models, Astron. J. 132 (2006) 2685

[65] J. Einasto and U. Haud, Galactic models with massive corona. I - Method. II - Galaxy, Astron. Astrophys. 223 (1989) 89

[66] M. Cappellari et al., The SAURON project - X. The orbital anisotropy of elliptical and lenticular galaxies: revisiting the $(\mathrm{V} / \mathrm{\text {, }})$ diagram with integral-field stellar kinematics, Mon. Not. R. Astron. Soc. 379 (2007) 418

[67] A. C. C. Guimarāes, L. Sodré, Density Profile, Velocity Anisotropy, and Line-of-sight External Convergence of SLACS Gravitational Lenses, Astrophys. J. 728 (2011) 33

[68] T. Treu, M. W. Auger, L. V. E. Koopmans, R. Gavazzi, P. J. Marshall, A. S. Bolton, The Initial Mass Function of Early-Type Galaxies, Astrophys. J. 709 (2010) 1195

[69] M. Cappellari et al., Systematic variation of the stellar initial mass function in early-type galaxies, Nature 484 (2012) 485

[70] A. A. Dutton, J. T. Mendel, L. Simard, Evidence for a non-universal stellar initial mass function in low-redshift high-density early-type galaxies, Mon. Not. R. Astron. Soc. 422 (2012) L33

[71] I. Jogensen, M. Franx and P. Kjaergaard, Spectroscopy for E and S0 galaxies in nine clusters, Mon. Not. R. Astron. Soc. 276 (1995) 1341

[72] E. Churazov et al., Comparison of approximately isothermal gravitational potentials of elliptical galaxies based on X-ray and optical data, Mon. Not. R. Astron. Soc. 404 (2010) 1165

[73] J. Wolf et al., Accurate masses for dispersion-supported galaxies, Mon. Not. R. Astron. Soc. 406 (2010) 1220

[74] A. V. Macciò, A. A. Dutton, F. C. van den Bosch, Concentration, spin and shape of dark matter haloes as a function of the cosmological model: WMAP1, WMAP3 and WMAP5 results, Mon. Not. R. Astron. Soc. 391 (2008) 1940

[75] R. Mandelbaum, U. Seljak and C. M. Hirata, A halo mass-concentration relation from weak lensing, JCAP 08 (2008) 006 
[76] D. H. Rudd, A. R. Zentner, A. V. Kravtsov, Effects of Baryons and Dissipation on the Matter Power Spectrum, Astrophys. J. 672 (2008) 19

[77] F. Governato, A. Zolotov, A. Pontzen, C. Christensen, S. H. Oh, A. M. Brooks, T. Quinn, S. Shen, J. Wadsley, Cuspy no more: how outflows affect the central dark matter and baryon distribution in $\Lambda$ cold dark matter galaxies, Mon. Not. R. Astron. Soc. 422 (2012) 1231

[78] A. V. Macciò, G. Stinson, C. B. Brook, J. Wadsley, H. M. P. Couchman, S. Shen, B. K. Gibson, T. Quinn, Halo Expansion in Cosmological Hydro Simulations: Toward a Baryonic Solution of the Cusp/Core Problem in Massive Spirals, Astrophys. J. 744 (2012) L9

[79] A. Toomre, J. Toomre, Galactic Bridges and Tails, Astrophys. J. 178 (1972) 623

[80] P. Ullio, L. Bergström, J. Edsjö and C. Lacey, Cosmological dark matter annihilations into $\gamma$ rays: A closer look, Phys. Rev. D 66 (2002) 123502

[81] M. Ackermann et al., Constraints on dark matter annihilation in clusters of galaxies with the Fermi large area telescope, JCAP 05 (2010) 025

[82] A. A. Abdo et al., Observations of Milky Way dwarf spheroidal galaxies with the Fermi-Large Area Telescope detector and constraints on dark matter models, Astrophys. J. 712 (2010) 147

[83] P. Prugniel and F. Simien, The fundamental plane of early-type galaxies: non-homology of the spatial structure, Astron. Astrophys. 321 (1997) 111

[84] I. Márquez, G. B. Lima Neto, H. Capelato, F. Durret and D. Gerbal, Gravo-thermal properties and formation of elliptical galaxies, Astron. Astrophys. 353 (2000) 873 